\title{
Coprophagy prevention alters microbiome, metabolism, neurochemistry, and cognitive behavior in a small mammal
}

\author{
Ting-Bei Bo $\mathbb{1}^{1,2} \cdot$ Xue-Ying Zhang $\mathbb{1}^{1,2} \cdot$ Kevin D. Kohl ${ }^{3} \cdot$ Jing Wen ${ }^{1,2} \cdot$ Shuang-Jie Tian ${ }^{1,2} \cdot$ De-Hua Wang $\mathbb{D}^{1,2}$
}

Received: 25 February 2020 / Revised: 15 June 2020 / Accepted: 25 June 2020 / Published online: 6 July 2020

(c) The Author(s), under exclusive licence to International Society for Microbial Ecology 2020

\begin{abstract}
Many small mammals engage in coprophagy, or the behavior of consuming feces, as a means to meet nutritional requirements when feeding on low-quality foods. In addition to nutritional benefits, coprophagy may also help herbivores retain necessary gut microbial diversity and function, which may have downstream physiological effects, such as maintaining energy balance and cognitive function. Here, we used collars to prevent Brandt's vole (Lasiopodomys brandtii) from engaging in coprophagy and monitored changes in microbial community structure, energy metabolism, and cognitive performance. In this research, we found that coprophagy prevention decreased alpha diversity of the gut microbiota, and altered proportions of microbial taxa such as Bacteroidetes, Firmicutes, and Oscillospira. Preventing coprophagy resulted in a reduced body mass, and increased food intake. Importantly, coprophagy prevention decreased vole cognitive behavior and altered levels of neurotransmitters in brain. Daily acetate administration was able to reverse some of the coprophagy prevention-induced changes in microbiota composition, metabolism, neurochemistry, and cognitive behavior. These findings identify the functional importance of coprophagy behavior and interactions between the gut microbiota, energy metabolism, and neurological function. Our results suggest that coprophagy contributes to stabilizing the gut microbiota, promoting microbial metabolism, maintaining host energy balance and, consequently, altering cognitive performance.
\end{abstract}

\section{Introduction}

The mammalian gut hosts a diverse community of microbes that have profound effects on host nutrition, physiology, and behavior [1]. The gut microbiota has been implicated in regulation of many physiological functions including aiding digestion, energy metabolism, and regulating the neuroendocrine system [2, 3]. Diverse gut microbiota

These authors contributed equally: Ting-Bei Bo, Xue-Ying Zhang

Supplementary information The online version of this article (https:// doi.org/10.1038/s41396-020-0711-6) contains supplementary material, which is available to authorized users.

De-Hua Wang

wangdh@ioz.ac.cn

1 State Key Laboratory of Integrated Management of Pest Insects and Rodents, Institute of Zoology, Chinese Academy of Sciences, Beijing 100101, China

2 CAS Center for Excellence in Biotic Interactions, University of Chinese Academy of Sciences, Beijing 100049, China

3 University of Pittsburgh, Pittsburgh, PA 15213, USA communities are especially important for herbivorous hosts, where they ferment indigestible dietary fibers into compounds that are more amenable to absorption and utilization by the host, such as short-chain fatty acids (SCFAs) [4, 5]. As a result, many mammalian herbivores acquire significant portions of their daily energy budgets from the processes of microbial fermentation $[6,7]$.

To access the more complex carbohydrates present in plants, disparate mammalian lineages expanded the size of foregut or hindgut regions as a means to lengthen gut retention times and facilitate bacterial fermentation [8]. To capitalize on the benefits provided by gut microbial communities, many hindgut-fermenting herbivores engage in coprophagy, or the behavior of consuming their own feces. This behavior allows hindgut-fermenting mammals to recover the nutrients that were liberated in the hindgut. Coprophagy is common in rodents, lagomorphs, and even primates [9], and is considered an adaptation to the metabolic disadvantages of small body size and feeding on lowquality foods $[10,11]$. The practice of coprophagy aids in the uptake of essential amino acids, vitamin B, and vitamin $\mathrm{K}$, SCFAs, and trace elements that are not fully absorbed, thus avoiding the loss of such nutrients [12]. Previous 
studies relying on culture-based methods have suggested that coprophagy may also help to stabilize the gut microbiome $[13,14]$, but to our knowledge this idea has not been tested in outbred rodents or using culture-independent methods.

Interactions between the gut microbiota and nutrition can have downstream impacts on cognitive function of animals $[15,16]$. Recent work demonstrates that gut microbiota play a central role in aspects of animal behavior and cognitive performance $[2,17,18]$. Gut microbes can elicit signals via the vagus nerve to the brain and vice versa [19-21]. Large numbers of studies have also demonstrated that the gut microbiota impacts nutrition and energy homeostasis $[22,23]$. So, the gut microbiota may affect the cognitive performance of animals directly through the gut-brain axis, or indirectly by affecting energy balance. Therefore, we hypothesized that coprophagy helps to stabilize the gut microbiota, promote microbial metabolism, maintain host energy balance and, consequently, contributes to host cognitive performance.

We tested this hypothesis in Brandt's vole (Lasiopodomys brandtii), a small mammalian herbivore distributed in the Inner Mongolia of China, Mongolia, and Baikal region of Russia. Brandt's voles need to consume large amounts of fiber to meet their energy needs, because of the shortage of high-nutrition food in the field [24]. Coprophagy can help Brandt's vole to obtain maximum energy yields from limited resources. Liu and Wang [10] reported that Brandt's vole readily engages in coprophagy behavior and has a rhythmic fecal intake (Brandt's voles ingested $68.8 \pm 7.4$ fecal pellets per day, averaging $17 \pm 2 \%$ of total feces produced). Daily feces volume of Brandt's vole can be calculated as is $18.3 \pm 2.2 \mathrm{~g} / \mathrm{Kg}^{0.67}$, and the food intake as $64.9 \pm 8.1 \mathrm{~g} / \mathrm{Kg}^{0.67}[11]$. We studied the relationship among coprophagy, the gut microbiota, energy metabolism, and cognitive performance, and specifically addressed three questions: (i) does the prevention of coprophagy behavior influence the community structure of the vole gut microbiota? (ii) Does prevention of coprophagy affect vole's metabolism and cognitive performance? (iii) If so, can provisioning of microbial products (specifically the most abundant short-chained fatty acid, acetate) rescue these phenotypes?

\section{Methods}

\section{Animals}

Brandt's voles for experiments were from laboratory colonies in the Chinese Academy of Sciences in Beijing. Voles were housed in groups (3-4 individuals per cage) in plastic cages $\left(30 \times 15 \times 20 \mathrm{~cm}^{3}\right)$ with sawdust bedding. For all experiments described below, voles were housed in animal rooms at $23 \pm 1^{\circ} \mathrm{C}$, with a photoperiod of 16Light: 8Dark. Our specific studies were carried out using adult male Brandt's voles (4-5 months old) with free access to water and food the commercial rabbit pellets (containing $18 \%$ protein, $3 \%$ fat, $12 \%$ fiber, and $47 \%$ carbohydrate, Beijing KeAo Bioscience Co.). For all experiments described below (Experiments 1, 2, and 3), animals were first moved to individual cages for a period of 2 weeks to acclimate to individual housing. For the entire duration of the experiments described below, animals were kept separately, in individual cages, so as to avoid microbial sharing across individuals [25].

\section{Experimental device}

We experimentally prevented coprophagy behavior using hard plastic collars. The collars were cut along a radial line and placed on the necks of the voles and are roughly $3-5 \%$ of the vole's body mass. For the "coprophagy prevention" treatment $(\mathrm{CP})$, the width of the collar was $1.5-2 \mathrm{~cm}$ and prevented animals from consuming their feces. During CP treatments, animals were placed in cages with wire bottoms, where animals did not have access to feces that fell through the screen. Through experiments, we determined that while wearing a collar, the mouth of the Brandt's vole cannot touch the anus, and the feces will fall under the metal mesh, and they cannot be picked up. Through preliminary experiments, we did not observe them eating feces, and the number and total mass of fecal pellets per day were significantly higher than the control group. However, these collars do not interfere with the animals' ability to eat or drink.

To control the potentially stressful effects of collars, we added a sham-coprophagy prevention (SCP) group, to control for the potentially stressful effects of wearing collars. Here, animals were fitted with sham-ring collars with a width of $0.5 \mathrm{~cm}$ (allowing animals to reach their anus and engage in coprophagy), and there was padding underneath the wire-screen, so that animals could access their feces.

\section{Experiment 1-does the prevention of coprophagy behavior influence the community structure of the vole gut microbiota?}

Before the experiment, eight male voles were moved into individual cages and acclimated for at least 2 weeks, during which they did not experience any treatments and could consume feces freely. At the beginning of the experiment, we collected fecal samples to inventory the baseline microbiome (0 week; Control), and placed voles in CP collars and wire-bottomed cages. After 2 weeks, we collected another fecal sample from each vole ( 2 weeks; CP 
treatment), and then removed collars and moved voles to individual, solid bottom cages for another 2 weeks, and collected additional fecal samples (4 weeks; Control). We repeated this process one more time to generate fecal samples for two more timepoints: 6 weeks; CP and 8 weeks; Control (Fig. S1a). After collection, all feces were stored at $-80{ }^{\circ} \mathrm{C}$ until DNA extraction and sequencing for microbial inventories (these methods are described in detail below).

\section{Experiment 2-does CP affect vole's metabolism and cognitive performance?}

Following acclimation to individual cages, eighteen male voles with similar body weight were randomly assigned into three groups (six animals in each group). A control group (Con) was not placed in any collars. The CP group was fitted with collars described above to prevent the ingestion of feces. Last, a SCP group of voles was placed in shortened collars described above to control for potentially stressful effects of the collars while still allowing voles to engage in coprophagy. Animals were held under these treatments for a total of 4 weeks (Fig. S1b). We measured body mass every two days. Starting body masses did not differ between the three groups (ANOVA: $F_{2,15}=0.016, p=0.98$ ). Food intake and fecal production (described below) were measured once per week. At the end of week 3, we assayed activity levels using the open-field test (described below). At the beginning of week 4, we measured resting metabolic rates (RMR) and non-shivering thermogenesis (NST) as measurements of host energy metabolism (one measurement per animal per day, in a random order; techniques described below). We then assayed cognitive function using a Y-maze (described below) at least 3 days after the last metabolic measurement was taken. Finally, voles were euthanized using $\mathrm{CO}_{2}$ and dissected. We collected serum and tissue samples to assay central genes and proteins involved in cognition, small intestine morphology, and concentrations of SCFAs in the cecum. These procedures are described in more detail below.

\section{Experiment 3-can provisioning of microbial products (specifically acetate) rescue these phenotypes?}

In this experiment, we tested whether supplementation of acetate (the most abundant SCFA in the vole gut) might rescue the alterations to the gut microbiome and host physiology induced by CP. Following acclimation, twenty-one male voles were divided into three groups: (i) a CP group gavaged with acetate $(\mathrm{CP}$-Ace $4.5 \mathrm{mmol} / \mathrm{d})$, dissolved in phosphate-buffered saline (PBS), (ii) a CP group gavaged with only phosphate-buffered saline (CP-PBS), and (iii) a control group gavaged with PBS $(\mathrm{CON})$. Animals were gavaged with about $0.2-\mathrm{ml}$ liquid twice per day. These three groups were housed individually for a total time of 6 weeks (Fig. S1c). We measured body mass every 2 days. Starting body masses did not differ between the three groups (ANOVA: $F_{2,18}=0.027, p=0.97$ ). Food intake and fecal production (described below) were measured once per week. During weeks 5 and 6, we measured aspects of behavior and cognitive performance using a Y-maze, a novel object test, and a conspecific recognition test (described below), with at least 3 days in between each assay. On the last day of week 6 , we collected feces at 9 a.m. and stored them at $-80{ }^{\circ} \mathrm{C}$ until DNA extraction and sequencing to inventory the gut microbiota. Finally, voles were euthanized using $\mathrm{CO}_{2}$ and dissected. We collected serum and tissue samples to assay central genes and proteins involved in cognition, small intestine morphology, and concentrations of SCFAs in the cecum. These procedures are described in more detail below.

\section{Measurement of body mass, food intake, and fecal production}

Body mass was measured around 9 a.m. using an electronic balance (Sartorius Model BL 1500, $\pm 0.1 \mathrm{~g}$ ). To measure food intake, voles were placed in a cage with a known amount of food $(\sim 50 \mathrm{~g})$. The following day, the amount of remaining food was measured, and food intake was calculated as the difference between these values. We measured fecal production by collecting all feces produced during a 24-h period, drying feces at $60^{\circ} \mathrm{C}$ for 1 week, and weighing feces using an electronic balance. Gross energy content of feces was determined using an IKA C2000 oxygen bomb calorimeter (IKA, Germany). Note that coprophagy typically occurs as animals consume feces directly from their anus, and so the periodic removal of feces (once per week) did not interfere with behavior of coprophagy.

\section{Measurement of RMR and NST}

We measured RMR, or the minimal amount energy consumed while animals are at rest. These measurements are generally considered as the baseline energetic costs of selfmaintenance, and an indicator for animals' energy metabolism [26, 27]. RMR values were measured as oxygen consumption using an open-circuit respirometry system (TSE labmaster, Germany). RMR were measured at $30{ }^{\circ} \mathrm{C}$ using $2.7 \mathrm{~L}$ metabolic chambers (Type I for mice), which we covered so that animals could not see one another. The air flow rate was $0.8 \mathrm{~L} / \mathrm{min}$. Oxygen consumption measurements were taken every $6 \mathrm{~min}$ for $3 \mathrm{~h}$. We took the average of the 3 lowest consecutive readings as the RMR.

We also measured the cost of NST, which is an important yet energetically costly mechanism for small mammals to 
increase heat production and maintain stable body temperatures [26]. These measurements were conducted at $25^{\circ} \mathrm{C}$ using a previously established method [28]. We injected animals subcutaneously with norepinephrine (NE) (Shanghai Harvest Pharmaceutical Co. Ltd) to elicit an elevated metabolic rate. The dosage of NE was calculated by the formula $\mathrm{NE}(\mathrm{mg} / \mathrm{kg})=2.53 \mathrm{BM}^{-0.4}$ for Brandt's voles. Voles were then placed in the respirometry equipment described above, and the cost of NST is estimated as the three highest consecutive readings of oxygen consumption during $1 \mathrm{~h}$ of measurement.

\section{Assays of activity, cognitive function, and behavior}

In Experiment 2, we used the open-field test as a general method for measuring animal's activity levels [29]. Voles were placed in the unfamiliar arena $(60 \mathrm{~cm}$ diameter with $45 \mathrm{~cm}$ high walls) for $5 \mathrm{~min}$ to adapt the environment. After this period of acclimation, their behavior was recorded for $10 \mathrm{~min}$ and analyzed using EthoVision Image Tracking System 20.0 (Noldus, Holland). We calculated the total moving distance traveled in $\mathrm{cm}$ over $10 \mathrm{~min}$. The apparatus for this behavioral test was cleaned thoroughly with $75 \%$ ethanol between uses to remove odor cues from previous individuals.

In Experiments 2 and 3, we assayed cognitive function using a Y-maze [30], which requires voles to use learning and memory to move toward a location that originally had food. The apparatus consisted of a white plastic maze with three arms ( $40 \mathrm{~cm}$ long, $30 \mathrm{~cm}$ high, and $8 \mathrm{~cm}$ wide) that intersect at $120^{\circ}$. The back wall of each arm was marked with different colored shapes. The voles began the test after a 12-h fasting period. First, we only opened two arms and put food in one arm, called the food arm (Fig. S1d). Voles were placed at one end of the "beginning" arm for $5 \mathrm{~min}$ to adapt and learn the location of the food and association with a colored shape marker. Voles were then removed from the Y-maze for $1 \mathrm{~h}$. Next, in the formal test, we removed the food and opened the third arm ("novel" arm), but did not change the markings on the walls (Fig. S1d). Thus, this method determined whether the animal could find the original location of the food using only the markings on the wall of the enclosure. Animals were allowed to move freely for $10 \mathrm{~min}$, and we calculated the distance traveled and amount of time spent in the "food" arm and the "novel" arm.

In Experiment 3, we assayed novelty-seeking and recognition behaviors by exposing animals to familiar and novel objects. Tests occurred in a covered plastic cage $\left(42 \times 27 \times 18 \mathrm{~cm}^{3}\right)$, and consisted of a 10-min training phase and a 5-min testing phase with a 1-h interval in between. Two plastic toys with different shapes (a plastic pyramid and a plastic cube) were used as the familiar object and novel object, respectively. During the 10-min training period, voles between each individual and each group tested were randomly placed in the cage and allowed to familiarize themselves with the pyramid toy (Fig. S1e). One hour later, the cube toy (defined as the novel object) was added to the cage (the positions of the two objects were random), and voles were subjected to a 5-min test (Fig. S1e). The order of voles during the testing phase was the same as that of training period, to ensure the same interval time between training and test. Preferences for the familiar and novel objects were measured as the duration and frequency of investigating each object during 5-min test. The toys and cage were wiped with $75 \%$ ethanol after each training and test.

In Experiment 3, we also investigated social interactions and conspecific recognition by repeatedly exposing voles to an unrelated individual, and then exposing them to a novel unrelated individual [31]. Each subject between each vole and each group tested was randomly placed into the experimental cage $\left(25 \times 45 \times 20 \mathrm{~cm}^{3}\right)$ and allowed to habituate for $10 \mathrm{~min}$. Thereafter, an unrelated male ("stimulus vole"; 30-40 days of age) was randomly introduced into the experimental cage for $5 \min (\operatorname{trial} 1(\operatorname{Tr} 1))$ and then removed. Thirty minutes later, the same stimulus vole was reintroduced for another $5 \mathrm{~min}(\operatorname{trial} 2(\operatorname{Tr} 2))$. This process was repeated once more to total three times $(\operatorname{Tr} 1, \operatorname{Tr} 2$, Trial 3 (Tr 3), Fig. S1f). During the fourth trial (New), a new, unrelated young was randomly introduced into the arena (Fig. S1f). This fourth trial tests the ability of voles to discriminate new conspecific individuals, and their cognitive flexibility. All 5-min interaction periods were video recorded; the frequency and duration of the subject's behaviors including olfactory investigation (i.e., sniffing of the conspecific's anogenital or head region) and close pursuit (i.e., closely following the conspecific) were quantified.

\section{Sample collection}

Prior to dissection, we collected blood samples via retroorbital bleeding, centrifuged samples at $4{ }^{\circ} \mathrm{C}(30 \mathrm{~min}, 1500 \mathrm{r})$, and froze the serum at $-80^{\circ} \mathrm{C}$. Animals were then sacrificed using $\mathrm{CO}_{2}$ between 9 a.m. and 11 a.m. We extracted the entire gastrointestinal tract (stomach, small intestine, cecum, and colon). Each section was laid flat to measure the length. Contents of each section were removed, tissues were rinsed five times with saline, and we then measured wet mass of each empty gut region. A small section from the middle of the small intestine was rinsed three times with $4 \%$ paraformaldehyde and then quickly put into a $4 \%$ paraformaldehyde solution and fixed for $24 \mathrm{~h}$. After routine paraffin embedding, a paraffin microtome was used to cut $5-\mu \mathrm{m}$ sections continuously, and 6-8 sections were placed horizontally on glass slides. Haemotoxylin and eosin 
staining of the gut was done as described elsewhere [32]. Then we measured the length and width of small intestinal villi using the ImageJ [33].

To collect brain tissues, we slightly modified previously published procedures $[34,35]$. We used dissection scissors to remove skin and open the skull, and then removed the entire brain into a glass dish over ice, ventral side up. We used ophthalmic scissors and curved forceps to carefully remove the hypothalamus from the other brain tissues. The hypothalamus was immediately homogenized in 9 parts saline $(0.9 \% \mathrm{NaCl})$, divided into two tubes, and frozen at $-80{ }^{\circ} \mathrm{C}$. Then, accessing the brain from the dorsal side, we used ophthalmic scissors and curved forceps to remove the cerebral cortex (isocortex) and expose the hippocampus tissues. The two hippocampi from each individual were frozen in separate tubes at $-80^{\circ} \mathrm{C}$ to be used in separate assays described below.

\section{Gut microbiota profiling}

Fresh feces and gut contents collected from the cecum were immediately frozen and stored at $-80^{\circ} \mathrm{C}$. DNA was extracted by $2 \times$ cetyltrimethyl ammonium bromide and phenol chloroform mixture (phenol: chloroform: isoamyl alcohol= 25:24:1), and was then isolated by a spin column using the SanPrep Column DNA Gel Extraction Kit (Sangon Biotec, 273 B518131-0100). Universal primers were used for PCR amplification of the V3-V4 hypervariable regions of $16 \mathrm{~S}$ rRNA genes and contained Illumina $3^{\prime}$ adaptor sequences as well as a 12-bp barcode [36]. Sequencing was pair-end on the Illumina HiSeq 2500 platform, with the sequencing strategy PE250. Raw sequencing reads were denoised, filtered according to barcode and primer sequences (Forward primer341F, CCTACGGGNGGCWGCAG; Reverse primer-805R, GACTACHVGGGTATCTAATCC), and classified with the Quantitative Insights Into Microbial Ecology (QIIME, version 1.9.1) software suite. The open-reference operation taxonomic unit (OTU) picking strategy in QIIME was used to identify OTUs, using a $97 \%$ sequence similarity. Resulting sequences were then searched against the Greengenes reference database of 16S rRNA gene sequences. We removed singleton OTUs. The OTU table was rarefied to a sequencing depth of 50,000 (Experiment 1) or 20,000 (Experiment 3) per sample for subsequent analyses of alpha and beta diversity. Alpha diversity metrics (Faith's phylogenetic diversity, Shannon index, and the number of observed OTUs) were calculated using rarefied OTU tables. To visually compare beta diversity metrics, we conducted nonmetric multidimensional scaling based on weighted and unweighted UniFrac distances. Variations in the beta diversity of bacterial communities were statistically compared using analysis of similarity (ANOSIM, permutations $=999$ ). Finally, we used the program PICRUSt (Phylogenetic Investigation of Communities by
Reconstruction of Unobserved States) to predict the functional components of the microbiome [37], and used the program STAMP to conduct $t$-tests comparing the abundances of predicted functions between 0 week and 2 weeks in Experiment 1 [38].

\section{Measurement of SCFAs}

For Experiments 2 and 3, we measured the concentrations of six SCFAs in the cecal contents collected at dissection: acetic acid, propionic acid, butyric acid, isobutyric acid, valeric acid, and isovaleric acid. Cecum contents were centrifuged, the supernatant was removed, and then treated with phosphoric acid for $3 \mathrm{~h}$ to stabilize SCFAs. Concentrations of SCFAs were measured by high-performance gas chromatography (GC) (Agilent 7890A; Agilent Technologies, Germany) with a GC autosampler and a FID system. Separations were performed in a $30 \mathrm{~m} \times 0.25 \mathrm{~mm} \times$ $0.25 \mu \mathrm{m}$ DB-WAX column (Agilent Technologies). Hydrogen gas (99.998\%) was used as carrier gas, with a flow rate of $1.0 \mathrm{~mL} / \mathrm{min}$. The system was operated at $250^{\circ} \mathrm{C}$. Injections were performed in the splitless mode at $230{ }^{\circ} \mathrm{C}$, and $0.5 \mu \mathrm{l}$ for each injection. The oven temperature was programmed from $60{ }^{\circ} \mathrm{C}(1 \mathrm{~min})$ at $5{ }^{\circ} \mathrm{C} / \mathrm{min}$ to $200^{\circ} \mathrm{C}$, then from $200{ }^{\circ} \mathrm{C}$ at $10{ }^{\circ} \mathrm{C} / \mathrm{min}$ to $230^{\circ} \mathrm{C}$. The total running time of each sample is $32 \mathrm{~min}$.

\section{Measurements of serum hormones}

We measured concentrations of hormones as indicators of energy metabolism. In Experiment 2, concentrations of triiodothyronine (T3) and thyroxine (T4) in serum were determined by radioimmunoassay using $\mathrm{I}^{125}$ RIA kits (Beijing North Institute of Biological Technology, Beijing, China). Intra- and inter-assay coefficients of variation were 2.4 and $8.8 \%$ for $\mathrm{T} 3$, and 4.3 and $7.6 \%$ for $\mathrm{T} 4$.

In Experiments 2 and 3, we measured serum ghrelin (the des-acylated form) using a Ghrelin EIA kit (Catalog No. EK-031-31, Phoenix Pharmaceuticals, USA). The minimum detected concentration by this kit was $0.13 \mathrm{ng} / \mathrm{mL}$. The intra- and inter-assay variations for ghrelin EIA were $10 \%$ and $15 \%$, respectively.

\section{Determination of monoamine neurotransmitters and turnover ratio (TR)}

We investigated changes in the concentrations of five neurotransmitters: NE, 3,4-dihydroxyphenylacetic acid (DOPAC), dopamine (DA), serotonin (5-HT), and 5hydroxyindoleacetic acid (5-HIAA). We used highperformance liquid chromatography with electrochemical detection. The internal standard was 3,4-dihydroxybelzyamine. We recorded the mass of hippocampus tissues 
$(\sim 0.01 \mathrm{~g})$ and calculated the mass of hypothalamic tissue in the saline homogenate $(\sim 0.015 \mathrm{~g})$. We homogenized samples in $200 \mu \mathrm{L}$ of cold perchloric acid $(0.1 \mathrm{M})$, conducted ultrasonic oscillation for $10 \mathrm{~s}$, and centrifuged samples at $16200 \mathrm{~g}$ at $4{ }^{\circ} \mathrm{C}$ for $30 \mathrm{~min}$. The resulting supernatant was filtered using a $0.2-\mathrm{mm}$ nylon filter. For measurements, the mobile phase $(\mathrm{pH}=4.0)$ consisted of $90-\mathrm{mM} \mathrm{NaH}_{2}-$ $\mathrm{PO}_{4} 2 \mathrm{H}_{2} \mathrm{O}, 50-\mathrm{mM}$ citric acid monohydrate, 1.7-mM alkylsulfonate (SAS), 50- $\mu \mathrm{M}$ EDTA, 2-mM NaCl, and $8 \%$ acetonitrile. Aliquots of tissue supernatants $(30 \mu \mathrm{l})$ were automatically injected into the detector (Thermo Scientific Ultimate 3000 RS Electrochemical Detector with 6041RS Ultra Amperometric Cell (6070.3000), highly efficient glassy carbon electrode (6070.3200), 6041RS sensor, and $\mathrm{Pd}$ reference electrode), which was set at $150 \mathrm{mV}$, and the column (AcclaimTM 120, C18 $3 \mathrm{~m} 120 \AA, 2.1 \times 150 \mathrm{~mm}$ ) was placed at $8{ }^{\circ} \mathrm{C}$. Compounds were identified by comparing their retention times with those of authentic reference compounds. The level of each monoamine was calculated by the internal standard method using Chromeleon 7 software (ThermoFisher Scientific, MA, USA).

\section{Quantitative calculation}

(1) The samples were quantified according to the correction factor + internal standard method.

Correction factor $(f)=$ (peak area of internal standard substance/concentration of internal standard substance)/ (peak area of standard/concentration of standard)

(2) Content (sample) $=f \times$ \{peak area of sample/(peak area of internal standard substance/concentration of internal standard substance) $\}$

(3) The amount of material was measured by $W$ (ng) = content (sample) $\times V$ (volume of liquid to be measured). Monoamine and metabolite levels are expressed as ug/g of wet tissue. Ratio of metabolite(s)/amine was calculated as an index of rate of metabolism; DOPAC/DA indicated DA turnover rate and 5-HIAA/5-HT indicated 5-HT turnover rate.

Many studies have indicated that the concentrations of neurotransmitter metabolites are useful indexes of neurotransmitter turnover [39]. By calculating the ratios of DOPAC/DA and 5-HIAA/5-HT, we were able to obtain an indication of DA and 5-HT utilization in tissue regions of the brain. Based on previous studies the TR for 5-HT was calculated from the equation $\mathrm{TR}=5$-HIAA content $/ 5-\mathrm{HT}$ content. The TR for DA was calculated from the equation $\mathrm{TR}=$ DOPAC content/DA content.

\section{Measurement of protein expression by Western blot}

Tissue samples of the hippocampus and saline homogenates of the hypothalamus were weighed, and homogenized in $200 \mu \mathrm{L}$ of radioimmunoprecipitation assay buffer (RIPA buffer: $10 \mathrm{mM}$ Tris, $158 \mathrm{mM} \mathrm{NaCl}, 1 \%$ TritonX-100, $5 \mathrm{mM}$ EDTA, $1 \mathrm{mM}$ DTT, $1 \mathrm{mM}$ PMSF, and 1:1000 parts of protease inhibitor cocktail [P8340, Sigma-Aldrich]). Homogenates were centrifuged at $16200 \mathrm{~g}$ at $4{ }^{\circ} \mathrm{C}$ for 30 min. Protein from the supernatant was placed in loading buffer (4\% SDS, $20 \%$ glycerol, $0.1 \%$ bromophenol blue, $250 \mathrm{mM}$ Tris $\mathrm{HCl} \mathrm{pH} 6.9,0.2 \%$ 2-beta mercaptoethanol) and denatured by heating at $100{ }^{\circ} \mathrm{C}$ for $3 \mathrm{~min}$. Total protein was separated by SDS-PAGE using a Mini Protean apparatus (BioRad Laboratories, PA, USA) then transferred to polyvinylidene fluoride membranes. Membranes were incubated for $12 \mathrm{~h}$ at $4{ }^{\circ} \mathrm{C}$ or $2 \mathrm{~h}$ at room temperature in $5 \%$ skim milk powder to reduce nonspecific antibody binding. The membranes were then exposed to primary antibodies (below) for $>12 \mathrm{~h}$ at $4{ }^{\circ} \mathrm{C}$. Then, membranes were exposed to appropriate secondary antibodies for $2 \mathrm{~h}$ at room temperature (either peroxidase-conjugated goat anti-rabbit IgG (111-035-003, Jackson) or peroxidase-conjugated goat antimice IgG (115-035-003, Jackson) depending on the primary antibody). Reaction products were visualized by chemiluminescence (ECL, Yesen). Protein was quantified with Lab image Software (BioRad, USA), expressed as relative units to housekeeping proteins.

In Experiment 2, we used whole tissue lysates of hippocampus and hypothalamus tissues to measure the expression of tyrosine hydroxylase (TH; AB152, Merck Millipore) and vasopression (AVP; AB1565, Merck Millipore), using GAPDH (A01020, Abbkine) as a housekeeping protein.

In Experiment 3 we used whole tissue lysates of hippocampus tissues to measure the expression of the following proteins: free fatty acid receptor 2 (FFAR2; ABC299, Merck Millipore), Neuronal Nuclei (NeuN; MAB377, Merck Millipore), glial fibrillary acific protein (GFAP; AB7260, Abcam), corticotropin releasing factor (CRF; AB184238, Abcam), CRF receptor (CRF1; AB95023, Abcam), AVP (AB1565, Merck Millipore), vasopressin receptor (AVP-R1; AB187753, Abcam), oxytocin (OT; AB911, Merck Millipore), OT receptor (AB217212, Abcam), proliferating cell nuclear antigen (PCNA; 13110, Cell Signaling Technology, Inc.), tyrosine kinase B (4603, Cell Signaling Technology, Inc.), and brain-derived neurotrophic factor (BDNF; AB203573, Abcam). For all these proteins, we used $\beta$-tubulin (A01030HRP, Abbkine) as a housekeeping protein. The characteristics and functions of biomarkers and neurotransmitters that we tested were shown (Table S1).

\section{Measurement of neuropeptides by real-time quantitative PCR (RT-qPCR)}

We assayed the influence of coprophagy prevention on the gene expression of agouti-related protein, neuropeptide $\mathrm{Y}$ (NPY), cocaine- and amphetamine-regulated transcript 
(CART), and pro-opiomelanocortin in the hypothalamus. We extracted total RNA from the hypothalamus using TRIzol agent, and then used reversed transcription to generate cDNA according to supplier specifications (Code No. RR820Q/A/B, TAKARA, Dalian, China). RT-qPCR analysis was carried out as follows: the cDNA samples $(2 \mu \mathrm{L})$ were used as a template for the subsequent PCR reaction using gene-specific primers (Table S2). The final reaction volume of $20 \mu \mathrm{L}$ contained 10 $\mu \mathrm{L}$ of $2 \times$ SYBR Premix EX Tag II (TAKARA), $0.4 \mu \mathrm{L}$ of $50 \times$ ROX Reference Dye, $2-\mu \mathrm{L}$ cDNA template, $0.8 \mu \mathrm{L}$ of forward primer and reverse primer (final concentration $0.4-\mu \mathrm{M}$ per primer), and $6-\mu \mathrm{L} \mathrm{dH}_{2} \mathrm{O}$. RT-qPCR was performed using Piko Real Software 2.2 (Piko Real 96, Thermo scientific, America). After an initial polymerase activation step at $95^{\circ} \mathrm{C}$ for $60 \mathrm{~s}$, amplification was followed by 40 cycles $\left(95^{\circ} \mathrm{C}\right.$ for $5 \mathrm{~s}, 55^{\circ} \mathrm{C}$ for $30 \mathrm{~s}$, and $72^{\circ} \mathrm{C}$ for $30 \mathrm{~s}$ ). The reaction was finished by the built-in melting curve. All samples were quantified for relative quantity of gene expression by using actin expression as an internal standard.

\section{Statistical analysis}

Statistical analysis was carried out using the SPSS 20.0 software package. Differences in body mass, food intake, and fecal production were compared between treatment groups using a repeated-measure ANOVA. Measurements on the final days of trials were compared using ANOVAs. Microbial measurements (proportions of taxa or functions, and alpha diversity metrics) were compared using one-way ANOVAs. Significance values were corrected for multiple measurements using the Benjamin-Hochberg FDR method [40]. We also compared the relative abundances of microbial genera using the pipeline LEfSe (linear discriminant analysis (LDA) effect size), using a LDA score threshold of 2 [41].

Beta diversity was compared using the ANOSIM test as described above. One-way ANOVA was used to compare measurements of RMR, NST, measurements of digestive anatomy, serum concentrations of hormones, SCFA concentrations measurements from behavioral trials, and expression of neurotransmitters. For all of these tests, post hoc differences between groups were conducted using the Student-Newman-Keuls method. All results were presented as means \pm SEM, and the level of statistical significance was set at $p<0.05$.

\section{Results}

\section{$\mathrm{CP}$ changes the gut microbiota composition}

We collected feces every 2 weeks during Experiment 1 to detect changes in the gut microbiota. Faith's phylogenetic diversity (alpha diversity) was lower during the coprophagy-prevention treatment (weeks 2 and 6) compared with the Control treatments (weeks 0,4 , and $8 ; F_{(4,35)}=$ $3.409, p=0.019$, Fig. 1a). The number of observed OTUs exhibited the same trend, while the Shannon index was not significantly different (Table 1). For beta diversity, analysis based on weighted and unweighted UniFrac distance showed significant differences among five sample types (ANOSIM, unweighted: $r=0.141, p=0.005$, weighted: $r=0.194, p=0.001$, Fig. 1b), and we can see that the samples collected during the CP period at (weeks 2 and 6) cluster together, while the other three timepoints, (when animals were experiencing the control treatment) also cluster together (Table S3).

We use repeated measures to analyze differences in the relative abundances of ten bacterial phyla: Actinobacteria, Bacteroidetes, Firmicutes, Verrucomicrobia, Cyanobacteria, Deferribacteres, Proteobacteria, Spirochetes, TM7, and Tenericutes (Table 2 and Fig. S2a). CP significantly decreased the relative abundances of Firmicutes $\left(F_{(4,35)}=\right.$ 9.446, $p<0.01)$ and increased the abundances of Bacteroidetes $\left(F_{(4,35)}=9.062, p<0.01\right.$, Fig. 1c). Specifically, when comparing between 0 week and 2 weeks, relative abundance of the phylum Firmicutes decreased from $60.1 \pm$ $2.5 \%$ at week 0 to $46.3 \pm 2.1 \%$ after 2 weeks of CP $(t=$ 4.254, $d f=14, p=0.001$; Fig. 1c). Relative abundances of Bacteroidetes increased from $35.1 \pm 2.5 \%$ at week 0 to $49.1 \pm 1.8 \%$ at week $2(t=4.46, d f=14, \quad p=0.001$; Fig. 1c). The other phyla showed no differences across time (Table 2 and Fig. S2b).

We analyzed the influence of $\mathrm{CP}$ on the microbiome at a finer scale by comparing the proportions of the top 30 most abundant OTUs (Fig. S2c). At the genus level, we compared 0-week group and 2 weeks group, and found Oscillospira, Rikenella, Anaerostipes, Oxalobacter, and Clostridium were significantly less abundant during $\mathrm{CP}$, while Prevotella and Dorea increased as a result of CP (LefSe, LDA $>2$, Table 3). The predicted functional potential of bacterial assemblages associated to each stool sample was predicted with PICRUSt using level 3 of Kyoto Encyclopedia of Genes and Genomes orthologs. The gut microbiome of samples collected during CP (2 weeks group) harbored significantly different functional categories when compared with samples collected during the control treatment ( 0 -week group) $(p<0.05$, Fig. 1d). Many of these different functional categories were related to carbohydrate metabolism (e.g., glycolysis/gluconeogenesis, starch, and sucrose metabolism), lipid metabolism (e.g., fatty acid biosynthesis), amino acid metabolism (e.g., Ala, asp, and glu acid metabolism), and cell signaling molecules (e.g., ABC transporters). 
a

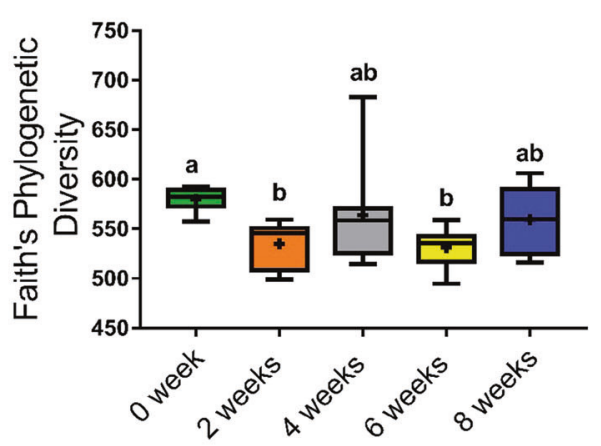

C
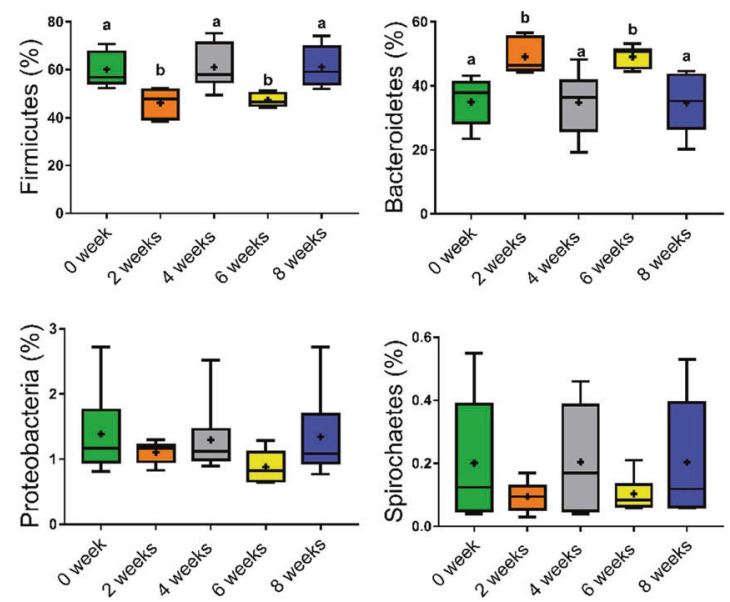

Fig. 1 Coprophagy affected the gut microbiota (Experiment 1). a Alpha diversity (Faith's phylogenetic diversity) of bacterial communities across groups ( $n=8$ per group, one-way ANOVA). b NMDS plot based on weighted and unweighted UniFrac distance metrics depicting the differences in fecal microbial community structure in different groups. c Relative abundances of bacterial phyla over the b
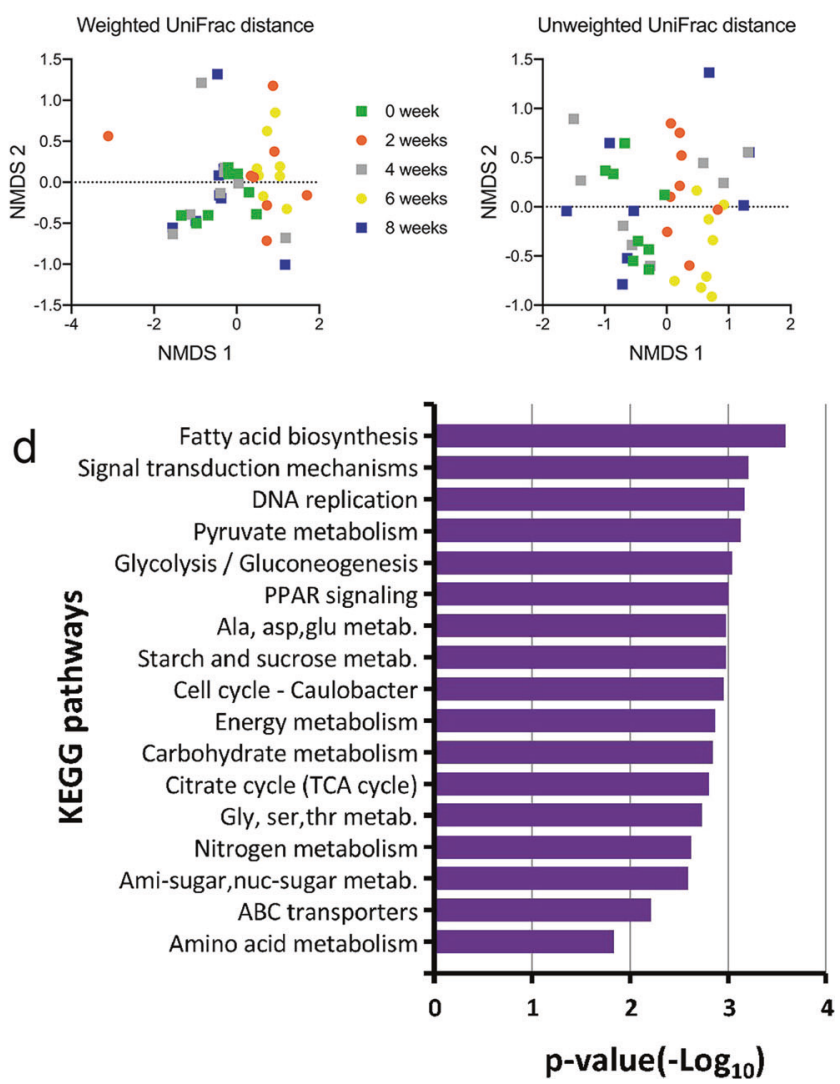

course of Experiment 1. d Predicted functional categories that differed significantly in relative abundance between 0 week and 2 weeks. Data are means $\pm \operatorname{SEM}$ ( $n=8$ per group), and bars that do not share the same letter are significantly different from one another based on the Student-Newman-Keuls method.
Table 1 The alpha diversity in Experiment 1 (one-way ANOVA).

\begin{tabular}{llll}
\hline Groups & Observed OTUs & Faith's phylogenetic diversity & Shannon \\
\hline 0 week (coprophagy) & $13797.6 \pm 135.2 \mathrm{a}$ & $579.8 \pm 4.0 \mathrm{a}$ & $10.5 \pm 0.05 \mathrm{a}$ \\
2 weeks (prevention) & $12461.3 \pm 223.7 \mathrm{a}$ & $535.0 \pm 8.17 \mathrm{~b}$ & $9.9 \pm 0.16 \mathrm{~b}$ \\
4 weeks (coprophagy) & $13452.4 \pm 610.7 \mathrm{a}$ & $564.01 \pm 18.6 \mathrm{ab}$ & $10.3 \pm 0.18 \mathrm{ab}$ \\
6 weeks (prevention) & $12599.13 \pm 158.8 \mathrm{a}$ & $531.3 \pm 7.1 \mathrm{~b}$ & $10.1 \pm 0.08 \mathrm{ab}$ \\
8 weeks (coprophagy) & $13211.9 \pm 300.7 \mathrm{a}$ & $559.4 \pm 11.7 \mathrm{ab}$ & $10.2 \pm 0.2 \mathrm{ab}$ \\
$F(4,35)$ & 2.878 & 3.409 & 2.36 \\
$p$ & 0.037 & 0.019 & 0.072 \\
\hline
\end{tabular}

Data were mean \pm SEM. The data is analyzed by one-way ANOVA with post hoc $(\mathrm{S}-\mathrm{N}-\mathrm{K}) . p<0.05$ indicates significant differences among groups. Different letters indicate significant differences between groups.

\section{Coprophagy affected the energy metabolism of animals}

Following $\mathrm{CP}$, body mass significantly decreased, despite the fact that the $\mathrm{CP}$ group exhibited significantly higher food consumption. Over the course of Experiment 2, the body mass in the Con group, $\mathrm{CP}$ group, and SCP group were not significantly different $\left(F_{(2,15)}=1.418, p=0.273\right.$, Fig. 2a), although there was a significant effect of time on body mass $\left(F_{(14,210)}=2.219, p=0.008\right.$, Fig. 2a). Body mass on the last day of the experiment was significantly different across the three treatment groups $\left(F_{(2,15)}=3.802\right.$, 
$p=0.046$ ), with the $\mathrm{CP}$ group having $15.4 \%$ lower body mass compared with the Control group. During $\mathrm{CP}$, food intake was significantly different across the three groups $\left(F_{(2,15)}=16.352, p<0.01\right.$, Fig. $\left.2 \mathrm{~b}\right)$, with CP group showing $44.7 \%$ higher food intake. Food intake did not differ depending on acclimation time $\left(F_{(4,60)}=9.112, p=0.163\right.$, Fig. 2b).

$\mathrm{CP}$ significantly altered the energy metabolism of voles. RMR were $28.2 \%$ lower in the $\mathrm{CP}$ group compared with the Con group $\left(F_{(2,15)}=4.956, p=0.022\right.$, Fig. $\left.2 c\right)$. Groups did not exhibit significant differences in energetic costs of NST $\left(F_{(2,15)}=3.176, p=0.071\right.$, Fig. 2d). Concentrations of thyroid hormone (T3) in the serum were significantly lower in the CP group when compared with the Con and SCP groups $\left(F_{(2,15)}=5.449, p=0.017\right.$, Fig. $\left.2 \mathrm{e}\right)$. Serum T4 concentration and the ratio of T3/T4 showed no group difference (Fig. S3a, b). We also found that CP increased animal activity measured by the open-field test, such that animals in the CP group traveled $47.9 \%$ more distance than the Con and SCP groups $\left(F_{(2,15)}=7.662, p=0.005\right.$, Fig. $\left.2 \mathrm{f}\right)$.

\section{$\mathrm{CP}$ alters digestive physiology}

We observed a marked increase in the mass of the cecum in the CP group compared with other groups $\left(F_{(2,15)}=7.819\right.$, $p=0.005$, Figs. $2 \mathrm{~g}$ and $\mathrm{S} 3 \mathrm{c})$. There was no differences across groups in terms of small intestine length $\left(F_{(2,15)}=\right.$ 1.953, $p=0.176$, Fig. S3d) or colon length or mass

Table 2 Repeated measures of proportions in phylum level in Experiment 1.

\begin{tabular}{llrllr}
\hline Phylum & \multicolumn{1}{l}{$p$} & Phylum & \multicolumn{1}{l}{$F$} \\
\hline Actinobacteria & 0.248 & 0.908 & Firmicutes & 9.446 & $<0.001$ \\
Bacteroidetes & 9.062 & $<0.001$ & Proteobacteria & 1.809 & 0.155 \\
Cyanobacteria & 0.213 & 0.929 & Spirochetes & 1.331 & 0.283 \\
Deferribacteres & 0.641 & 0.638 & TM7 & 2.416 & 0.072 \\
Verrucomicrobia & 2.405 & 0.073 & Tenericutes & 1.255 & 0.311 \\
\hline
\end{tabular}

Data were mean \pm SEM. $p<0.05$ indicates significant differences among groups.
(Fig. S3e, f). To further investigate the changes in the intestinal morphology, we measured the intestinal villus length and crypt depth, and found that villus length was significantly decreased in the CP group $\left(F_{(2,15)}=5.519\right.$, $p=0.016$, Fig. $2 \mathrm{~h}$ ), with no significant differences in crypt depths. The mass of feces recovered per day was higher in the CP group than Con and SCP group $\left(F_{(2,15)}=5.76, p=\right.$ 0.014, Fig. 2i). The total calories of feces in CP was higher than Con and SCP group $\left(F_{(2,15)}=6.183, p=0.011\right.$, Fig. 2 j), though energy density of feces did not differ across groups.

We measured concentrations of several SCFAs, which are associated with gut microbiota activity. Specifically, CP significantly decreased the concentrations of acetic acid $\left(F_{(2,15)}=6.273, p=0.01\right.$, Fig. $\left.2 \mathrm{k}\right)$, propionic acid $\left(F_{(2,15)}=\right.$ 15.337, $p<0.01$, Fig. $2 \mathrm{k})$, and butyric acid $\left(F_{(2,15)}=6.396\right.$, $p=0.01$, Fig. $2 \mathrm{k}$ ), suggesting that $\mathrm{CP}$ decreases fermentation activity of the gut microbiome. Concentrations of isobutyric acid, isovaleric acid, and valeric acid were not altered by CP (Fig. 21). The ratio of acetic acid to butyric acid exhibited a nonsignificant trend across the three groups $\left(F_{(2,15)}=3.315, p=0.064\right)$, with the CP group exhibiting a slightly higher acetate: butyrate ratio.

\section{CP decreased cognitive performance and alters brain chemistry}

In the $\mathrm{Y}$-maze, voles in the $\mathrm{CP}$ group spent significantly less time in the "food" arm compared with the other groups $\left(F_{(2,15)}=5.146, p=0.02\right.$. Fig. 3a), suggesting impairment in spatial learning and memory. The proportion of distance traveled in food arm was not different among three groups $\left(F_{(2,15)}=0.188, p=0.831\right.$, Fig. 3a). Similarly, activity levels (as measured by the total distance traveled in the Ymaze) did not exhibit significant differences across groups $\left(F_{(2,15)}=0.244, p=0.786\right.$, Fig. 3b).

We investigated potential changes in concentrations of monoamine neurotransmitters across the three groups, as these neurotransmitters have been associated with social behavior and cognition. $\mathrm{CP}$ did not alter the levels of $\mathrm{NE}$ in
Table 3 Different OTUs between 0 -week group and 2 weeks group in Experiment 1.

\begin{tabular}{lllll}
\hline Feature & Logarithm value & Group & LDA & $p$ \\
\hline Lachnospiraceae.g__Anaerostipes & 1.92 & 0 week & 3.59 & 0.05 \\
Ruminococcaceae.g_Oscillospira & 4.12 & 0 week & 3.41 & 0.03 \\
Clostridiaceae.g__lostridium & 3.45 & 0 week & 2.87 & 0.03 \\
Rikenellaceae.g_Rikenella & 2.45 & 0 week & 3.40 & 0.04 \\
Oxalobacteraceae.g_Oxalobacter & 1.77 & 0 week & 3.79 & 0.04 \\
Lachnospiraceae.g_Dorea & 2.50 & 2 weeks & 3.32 & 0.04 \\
Prevotellaceae.g__Prevotella & 4.09 & 2 weeks & 3.63 & 0.00 \\
\hline
\end{tabular}

The table listing the feature is discriminative, the logarithm value of the highest mean among all the classes, the class with the highest mean, and the logarithmic LDA score. 
a

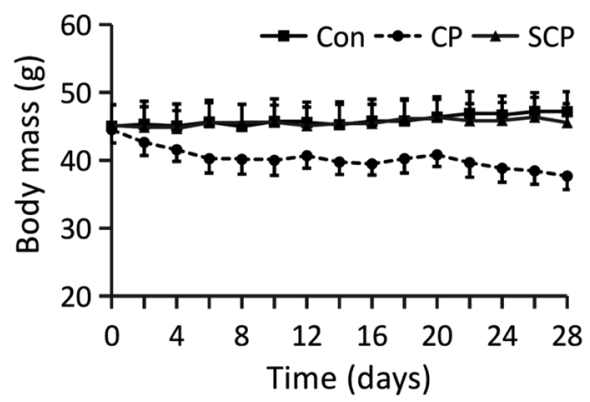

d

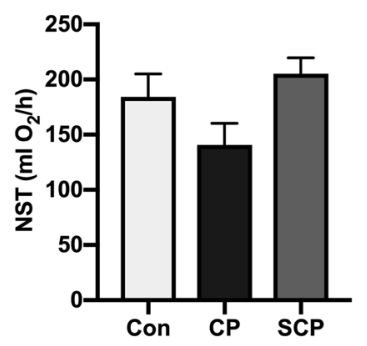

$\mathrm{h}$

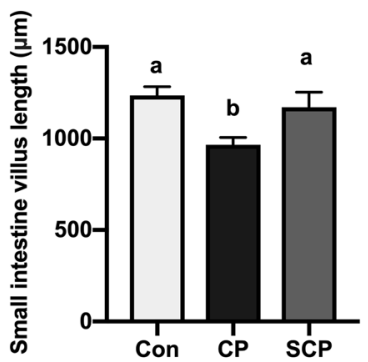

i b

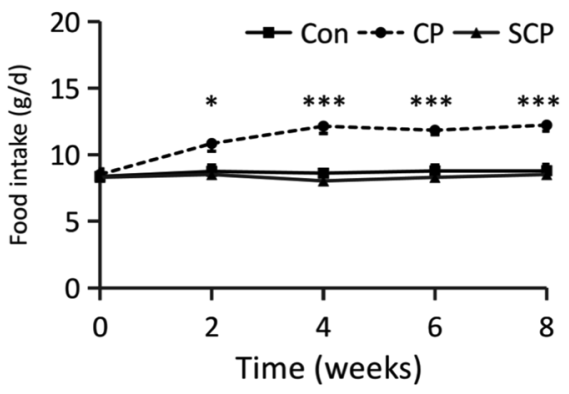

f
C

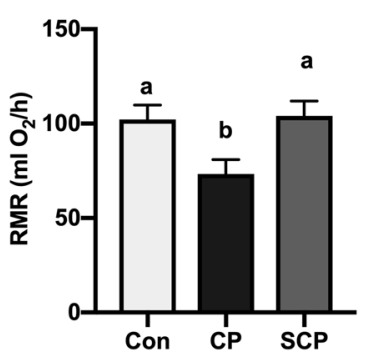

g

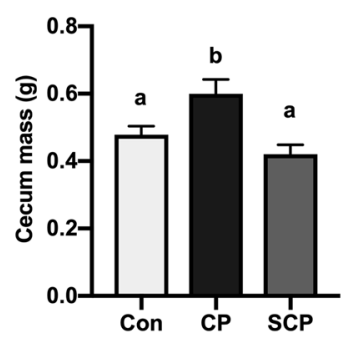

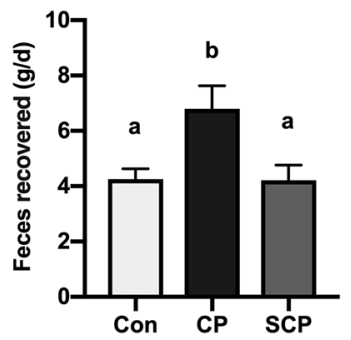

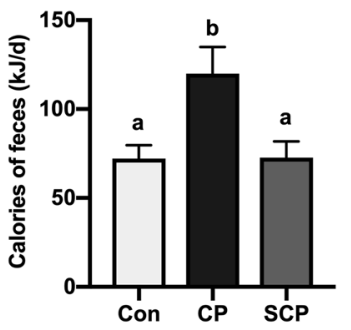

k

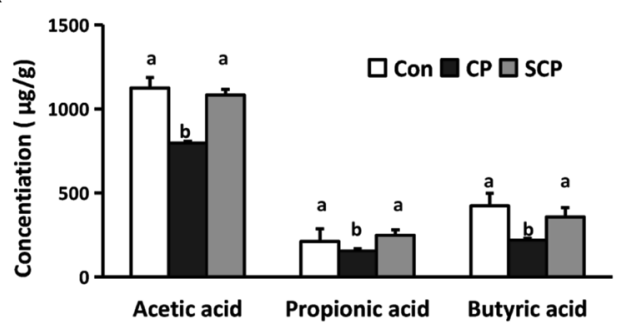

Fig. 2 Coprophagy prevention induces alterations in metabolic phenotypes in voles (Experiment 2). a Changes of body mass over the length of the experiment. b Changes of food intake over the length of the experiment (repeated measures ANOVA). $\mathbf{c}$ Resting metabolic rates (RMR) of treatment groups. d Cost of non-shivering thermogenesis (NST) of treatment groups. e Concentration of T3 in serum. f Moving distance in three groups in the open-field test. $\mathbf{g}$ Differences among three groups in cecum mass. $\mathbf{h}$ Small intestinal villus length.

the hypothalamus $\left(F_{(2,15)}=1.571, p=0.24\right.$, Fig. $\left.3 c\right)$ or hippocampus $\left(F_{(2,15)}=1.566, p=0.241\right.$, Fig. $\left.3 d\right)$. CP reduced the concentration of DA both in the hypothalamus (Fig. 3c) and hippocampus $\left(F_{(2,15)}=6.110, p=0.011\right.$, Fig. 3d), and reduced the concentration of DOPAC in the

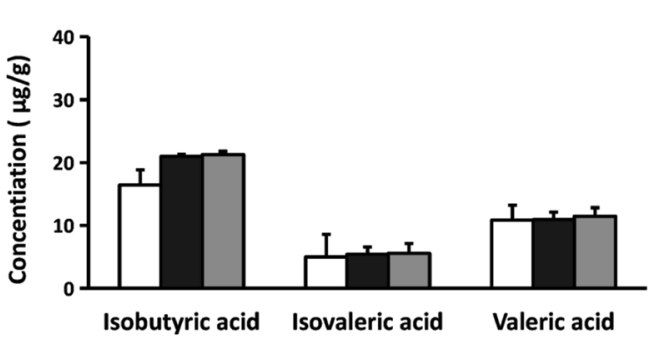

i The total mass of feces recovered per day at the end of the experiment. $\mathbf{j}$ The total caloric content of recovered feces per day. $\mathbf{k}$, $\mathbf{l}$ The concentrations of six short-chain fatty acids (SCFAs) in cecum contents. Con: control group, coprophagy, CP: coprophagy prevention, SCP: coprophagy with a sham collar. Data are presented as means $\pm \operatorname{SEM}$ ( $n=6$ per group), and bars that do not share the same letter are significantly different from one another based on the Student-Newman-Keuls method.

hypothalamus $\left(F_{(2,15)}=4.067, p=0.039\right.$, Fig. 3c). Similarly, concentrations of 5-HT and its metabolite 5-HIAA in the hippocampus of $\mathrm{CP}$ voles were lower than the Con and SCP groups $\left(F_{(2,15)}=4.017, p=0.04 ; F_{(2,15)}=4.167\right.$, $p=0.036$, Fig. $3 \mathrm{~d}$ ). The TRs of 5-HT and DA did not 

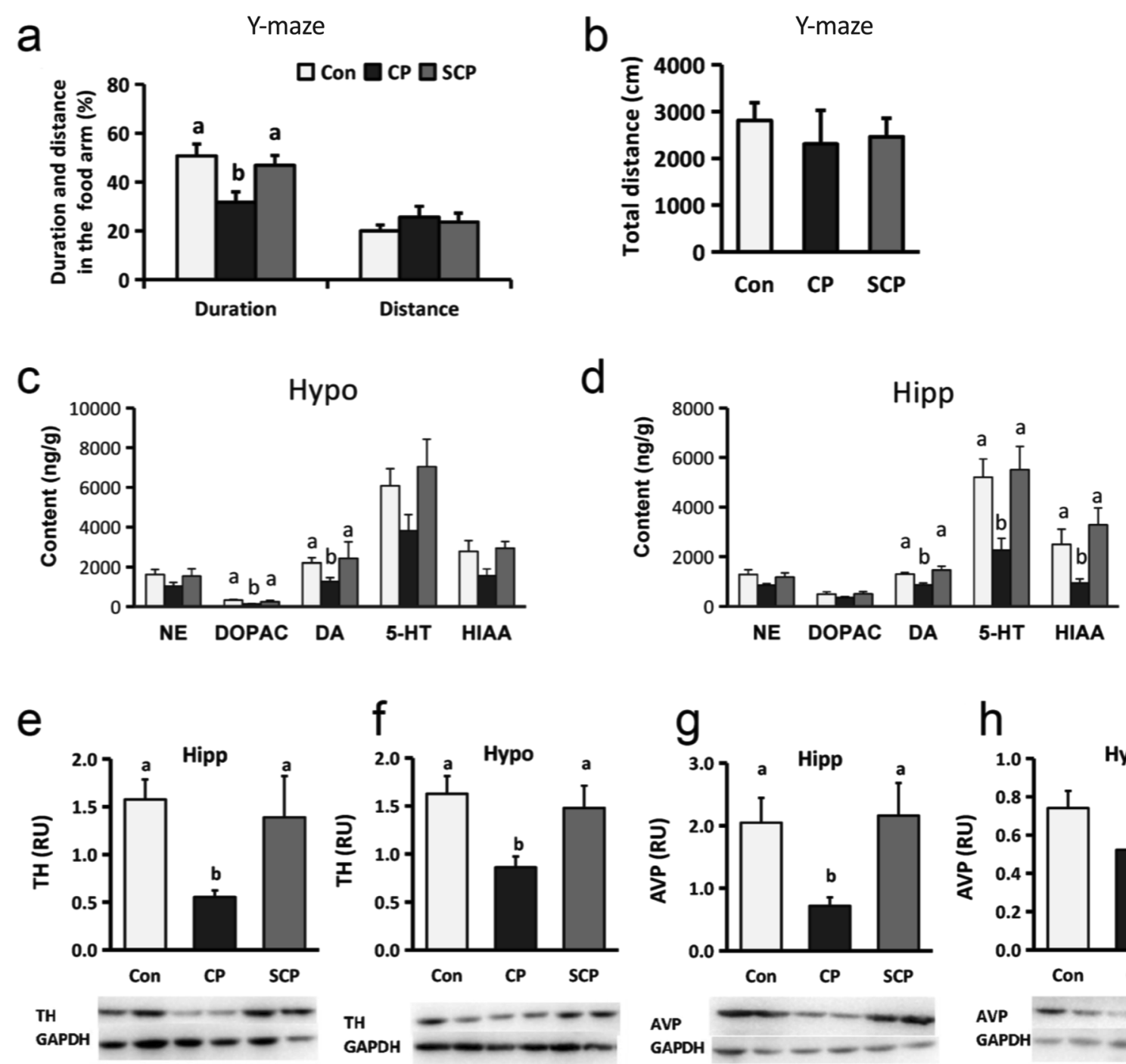

Fig. 3 Coprophagy prevention induces alterations in cognitive performance and neurodevelopment in voles (Experiment 2). a The duration and distance in the "food arm" during the Y-maze test. b The total moving distance of three groups in the Y-maze test. c The concentration of neurotransmitters in hypothalamus. $\mathbf{d}$ The concentration of neurotransmitters in the hippocampus. e, $\mathbf{f}$ Expression of tyrosine
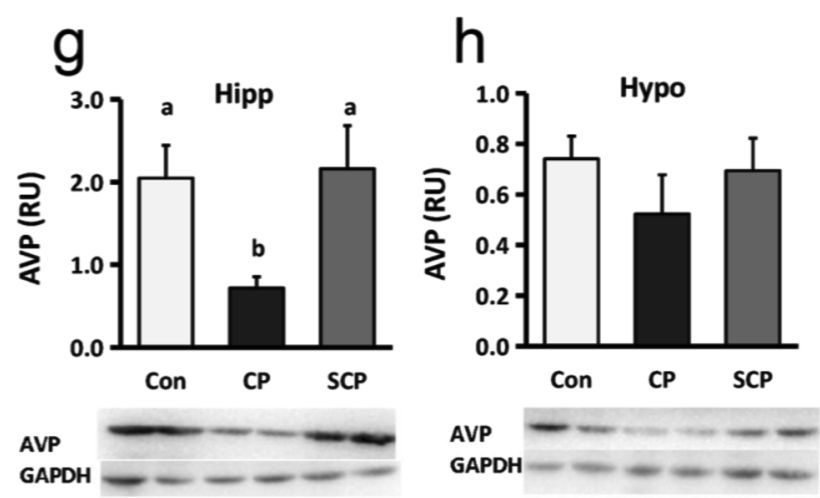

exhibit significant differences among three groups $(p>0.05$, Fig. S3g, h).

Last, we found that $\mathrm{CP}$ reduced the expression of $\mathrm{TH}$ in the hippocampus $\left(F_{(2,15)}=8.338, p=0.004\right.$, Fig. 3e $)$ and hypothalamus $\left(F_{(2,15)}=4.903, p=0.023\right.$, Fig. $\left.3 \mathrm{f}\right)$. In addition, $\mathrm{CP}$ reduced expression of AVP in the hippocampus (AVP; $F_{(2,15)}=4.59, p=0.028$, Fig. $3 \mathrm{~g}$ ), but not in the hypothalamus $\left(F_{(2,15)}=0.803, p=0.466\right.$, Fig. $\left.3 \mathrm{~h}\right)$.

\section{Supplementation with acetate reversed changes in host physiology induced by CP}

In Experiment 3, we supplemented CP animals with acetate to investigate whether phenotypes were rescued. We chose to

hydroxylase (TH) in hypothalamus and hippocampus. $\mathbf{g}$, $\mathbf{h}$ Expression of arginine vasopressin (AVP) in the hypothalamus and hippocampus. Con: control group, coprophagy, CP: coprophagy prevention, SCP: copropghay with a sham collar. Data are presented as means \pm SEM $(n=6$ per group), and bars that do not share the same letter are significantly different from one another based on the Student-Newman-Keuls method.

focus on acetate in our studies, as it is the most abundant of the SCFA in the feces. Body mass did not differ significantly across our three groups (Time: $F_{(19,323)}=6.353, p<0.001$; Group: $F_{(2,17)}=0.035, p=0.965$; Fig. 4a). Daily acetate gavage partially attenuated $\mathrm{CP}$-induced increases in food intake, such that food intake of the CP-Ace group was intermediate between the Con and CP-PBS groups (whole model: Time: $F_{(5,90)}=9.903, p<0.001$; Group: $F_{(2,18)}=$ 5.260, $p=0.016$; analyses at specific timepoints: 0 week: $F_{(2,18)}=0.124, p=0.884 ; 1$ week: $F_{(2,18)}=5.941, p=0.012$; weeks: $F_{(2,18)}=4.003, p=0.036 ; 3$ weeks: $F_{(2,18)}=5.559$, $p=0.013$; 4 weeks: $F_{(2,18)}=5.261, P=0.016 ; 5$ weeks: $F_{(2,18)}=7.911, p=0.003$, Fig. 4b, S4a). Alterations to the length of small intestine $\left(F_{(2,18)}=3.748, \quad p=0.044\right.$, 
a

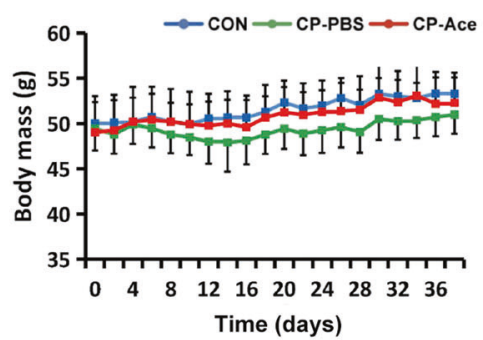

d

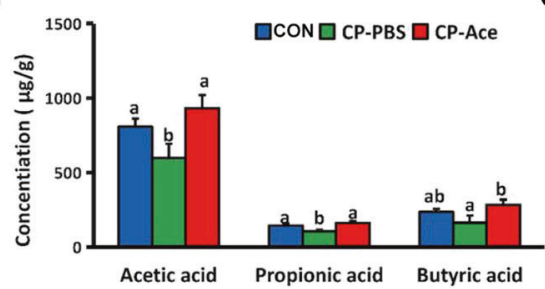

g

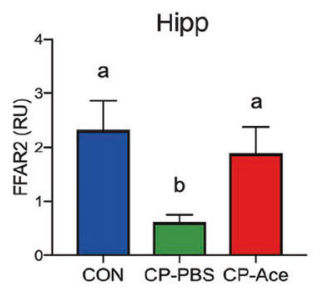

FFAR2

$\beta$-Tubulin

h b

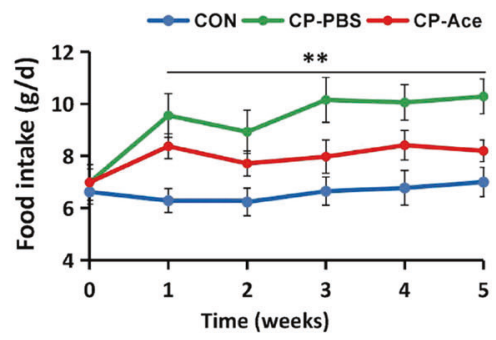

e

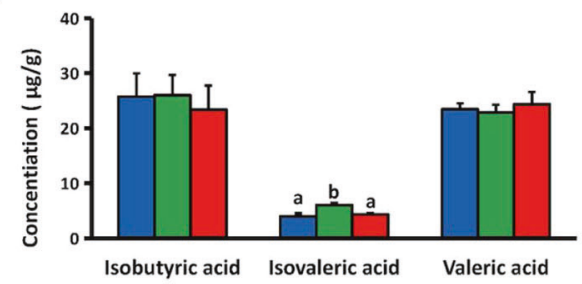

C

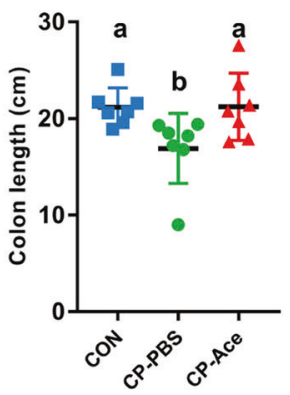

f

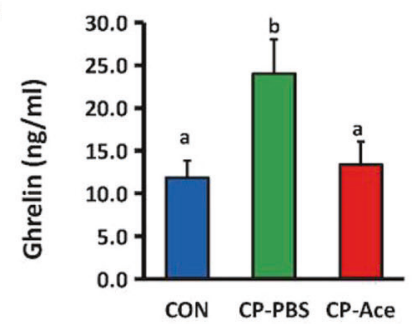

$\square$ CON $\square$ CP-PBS $\square$ CP-Ace

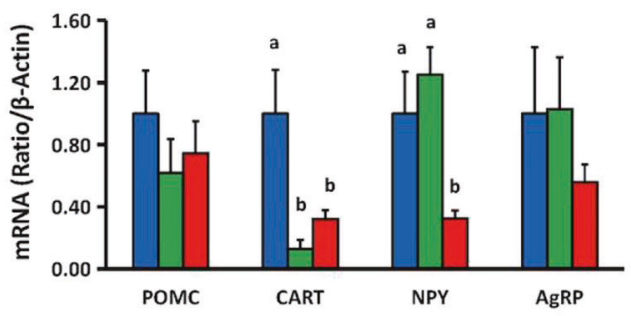

i
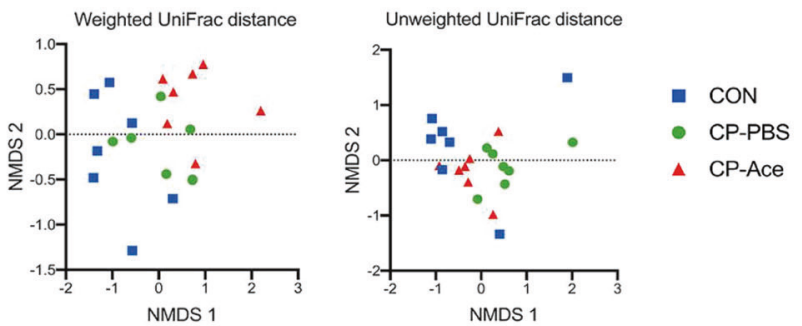

j

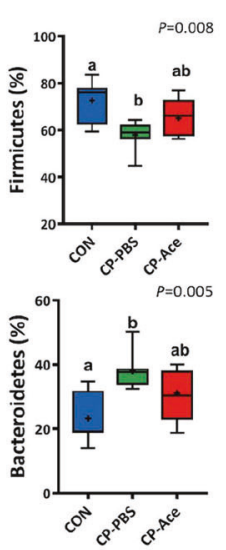

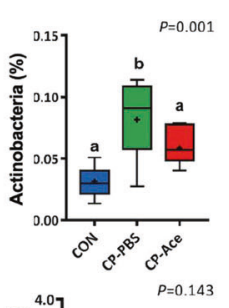

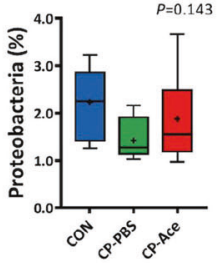

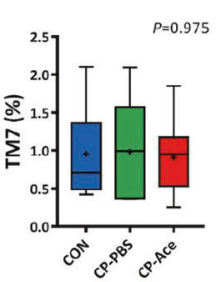

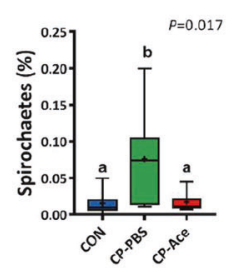

Fig. 4 Effects of acetate supplementation on host physiology and gut microbiota during coprophagy prevention (Experiment 3). a Changes of body mass over the length of the experiment. b Changes of food intake over the length of the experiment (repeated measures ANOVA). c Colon lengths of the three treatment groups. d, e The concentrations of six short-chain fatty acids (SCFAs) in cecal contents. f Ghrelin concentration in the serum. $\mathbf{g}$ Expression of FFAR2 in the hippocampus. $\mathbf{h}$ Expression of neuropeptides in hypothalamus. (POMC, CART, NPY, AgRP). i NMDS plot based on weighted and unweighted UniFrac distance metrics representing the differences in fecal microbial community structure in different groups. $\mathbf{j}$ Relative abundances of microbial phyla across treatment groups. CON: control group, coprophagy, CP-PBS: coprophagy prevention with PBS gavage, CP-Ace: copropghay prevention with Acetate gavage. Data are means \pm SEM ( $n=7$ per group), and bars that do not share the same letter are significantly different from one another based on the Student-Newman-Keuls method. 
Fig. S4b, c) and colon $\left(F_{(2,18)}=4.387, p=0.028\right.$, Fig. $\left.4 c\right)$ that were induced by $\mathrm{CP}$ were ameliorated by daily acetate supplementation. Colon morphology and the length and width of colon villi also recovered after acetate supplementation (Fig. S4d).

Daily supplementation of acetate normalized concentrations of SCFAs (Fig. S4e). Because we added acetate artificially, the acetate content in CP-Ace group was similar to the CON group, with both of these groups being higher than the CP-PBS group $\left(F_{(2,18)}=4.340, p=0.029\right.$, Fig. $\left.4 d\right)$. Similarly, concentrations of both propionic acid $\left(F_{(2,18)}=\right.$ 5.804, $p=0.011$, Fig. 4d) and isovaleric acid increased $\left(F_{(2,18)}=7.176, \quad p=0.005\right.$, Fig. $\left.4 \mathrm{e}\right)$ and were similar between the CON and CP-Ace groups, and significantly different from the CP-PBS group. There was no difference in the ratio of acetic acid to butyric acid between the three groups $\left(F_{(2,18)}=2.515, p=0.109\right)$.

Daily acetate supplementation reduced serum ghrelin concentrations of the CP-Ace group to match that of the Con group, while the CP-PBS group exhibited higher concentrations than the other two groups $\left(F_{(2,18)}=4.828\right.$, $p=0.021$, Fig. 4f). In the hippocampus, there are FFAR2 receptors that respond to SCFAs. CP induced significant decreases in the expression of FFAR2, while acetate supplementation rescued the expression of FFAR2 to match that of the control group $\left(F_{(2,18)}=4.493, p=0.026\right.$, Fig. 4g).

However, acetate supplementation did not ameliorate all physiological differences. The expression of NPY was significantly lower in the CP-Ace group compared with the other groups $\left(F_{(2,15)}=4.588, p=0.028\right.$, Fig. 4 h $)$. CART, a neuropeptide that inhibits appetite, was more highly expressed in the Control group than the CP-PBS or CP-Ace groups $\left(F_{(2,15)}=7.236, p=0.006\right.$, Fig. $\left.4 \mathrm{~h}\right)$.

\section{Supplementation of acetate can change the gut microbiota}

Alpha diversities of three treatment groups were not significantly different (Table S4). For the beta diversity, analysis based on unweighted UniFrac distance showed significant differences among the three groups (ANOSIM, three groups: unweighted: $r=0.198, p=0.004$; weighted: $r=0.341, p=0.001)$ though pairwise comparisons were not statistically significant (Table S5 and Fig. 4i).

Supplementation with acetate rescued the abundances of several microbial groups (Fig. S4f). Abundances of the phylum Firmicutes were lower in the CP-PBS group compared with CON $\left(F_{(2,18)}=6.297, p=0.008\right.$, Fig. 4 j) and abundance of the phylum Bacteroidetes was higher in the CP-PBS group compared with the Control group $\left(F_{(2,18)}=7.297, p=0.005\right.$, Fig. $\left.4 \mathrm{j}\right)$; abundances of these phyla were intermediate in the CP-Ace group. The relative abundance of Spirochetes was significantly increased in the CP-PBS group and abundances were recovered by supplementation of acetate $\left(F_{(2,18)}=5.114, p=0.017\right.$, Fig. 4j). Acetate supplementation did not reverse the effect of $\mathrm{CP}$ on Actinobacteria $\left(F_{(2,18)}=9.835, p=0.001\right.$, Fig. $4 \mathrm{j}$ ).

To assess how CP and supplementation with acetatealtered predicted functions of microbial communities, we applied LEfSe method with LDA score $>2$ to PICRUSt results (Fig. S4g). Many functional categories like carbohydrate metabolism (e.g., Carbohydrate metabolism, Pyruvate metabolism, and Galactose metabolism), amino acid metabolism (e.g., Amino sugar and nucleotide sugar metabolism) were not affected by acetate supplementation, because there was no difference between CP-PBS voles and CP-Ace voles (Fig. S4h).

\section{Supplementation of acetate can rescue cognitive behavior during $\mathrm{CP}$}

To test whether microbial metabolites may influence aspects of animal cognitive performance during $\mathrm{CP}$, we conducted a series of behavioral tests with daily acetate supplementation. The results of Y-maze test showed that there was no difference in the total moving distance across the three groups $\left(F_{(2,18)}=1.579, p=0.233\right)$. However, acetate supplementation rescued behaviors, such that the $\mathrm{CON}$ and CP-Ace groups both traveled a greater total distance $\left(F_{(2,18)}=6.963\right.$, $p=0.006$, Fig. 5a) and proportion of distance $\left(F_{(2,18)}=\right.$ $3.627, p=0.047$, Fig. $5 b)$ in the food arm when compared with the CP-PBS group. There was no difference among the three groups in the proportion of time spent in the food arm $\left(F_{(2,18)}=1.065, p=0.366\right.$, Fig. 5b) .

In the object recognition test, animals explore novel objects by quick approaches to sniff and investigate novel items, typically followed by more prolonged investigations next to novel objects. Supplementation with acetate reversed the behavioral changes induced by $\mathrm{CP}$. The $\mathrm{CON}$ and CP-Ace groups had both a higher frequency of investigating the novel object $\left(F_{(2,18)}=7.107, p=0.005\right.$, Fig. $\left.5 c\right)$ and a higher percentage of residence time with the novel object $\left(F_{(2,18)}=4.376, p=0.028\right.$, Fig. $\left.5 \mathrm{~d}\right)$ when compared with the CP-PBS group. Another way to interpret this data is to investigate the preferences for familiar versus novel objects within a treatment group. Both the Con and CP-Ace groups exhibited a significantly greater frequency of investigations toward the novel object $(t=2.277, d f=12$, $p=0.042 ; t=2.153, d f=12, p=0.05$, Fig. $5 c$ ) , and significantly more time with the novel object $(t=2.769, d f=$ $12, p=0.017 ; t=2.249, d f=12, p=0.044$, Fig. 5d). However, the CP-PBS group did not seem to differentiate these two objects, as this did not exhibit differences in the frequency of investigation $(t=0.15, d f=12, p=0.883$, 

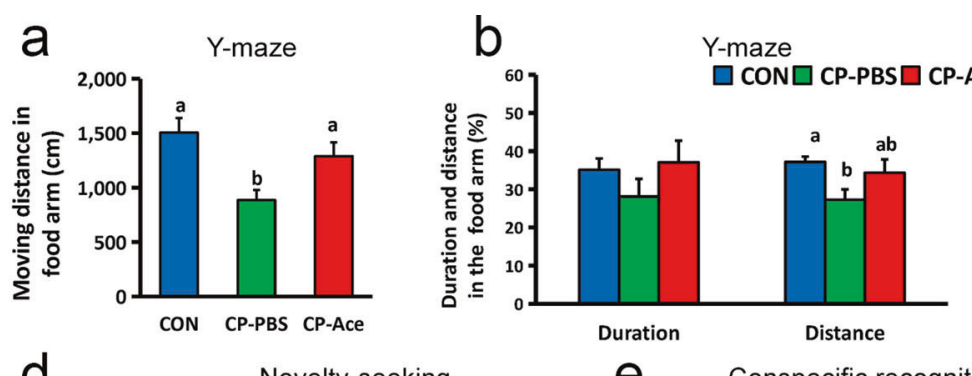

d Novelty-seeking

e Conspecific recognition
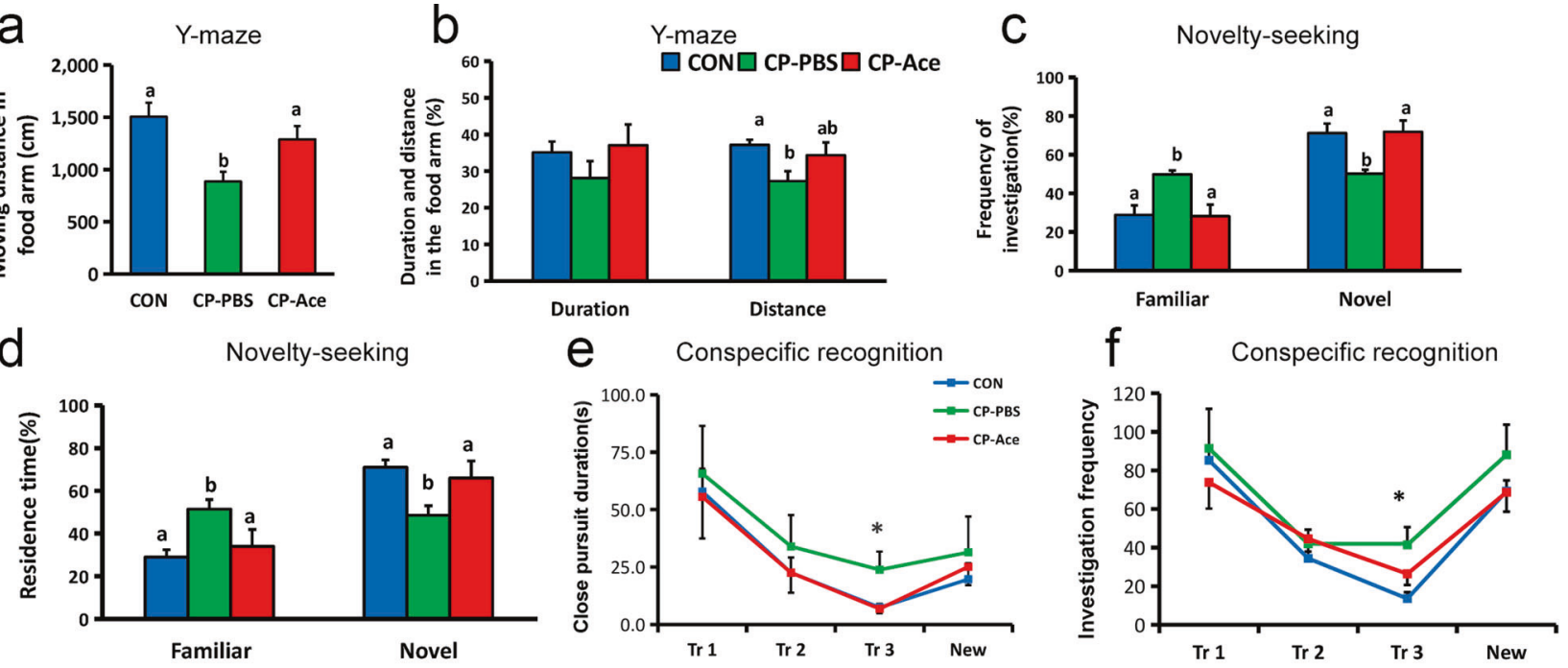

9
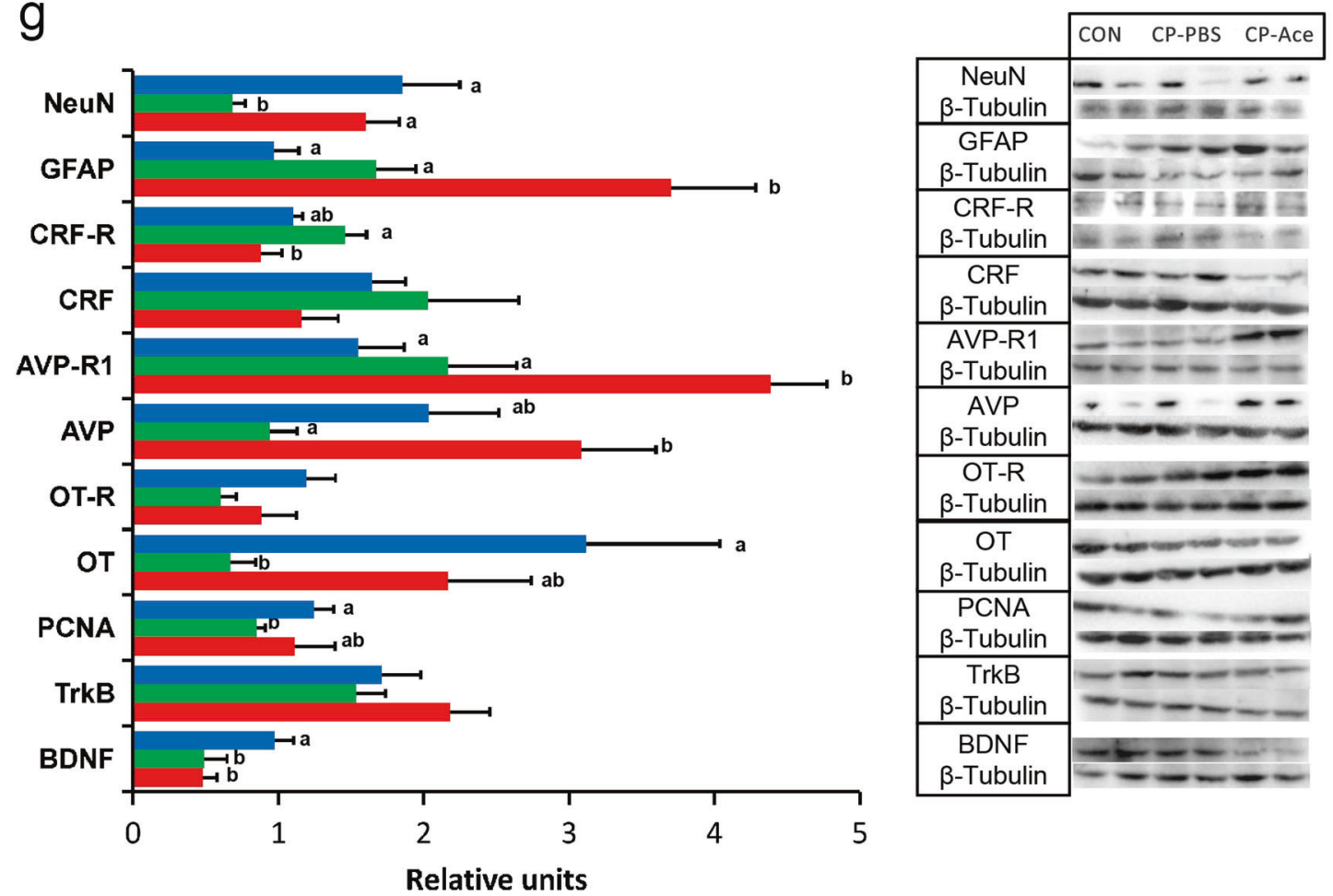

Fig. 5 Supplementation of acetate can promote the cognitive performance and development of hippocampal neurons during coprophagy prevention (Experiment 3). a The moving distance in the "food arm" during the Y-maze test. b The proportion of time and distance in the "food arm" during the Y-maze test. $\mathbf{c}$ The proportion of investigations of the familiar and novel objects. $\mathbf{d}$ The proportion of residence time with familiar and novel objects. e The time spent in

close pursuit of conspecifics. $\mathbf{f}$ The frequency of investigation of conspecifics. $\mathrm{g}$ The expression of neuropeptides in the hippocampus. CON: control group, coprophagy, CP-PBS: coprophagy prevention with PBS gavage, CP-Ace: copropghay prevention with acetate gavage. Data are means $\pm \operatorname{SEM}(n=7$ per group), and bars that do not share the same letter are significantly different from one another based on the Student-Newman-Keuls method.

Fig. 5c) or in the proportion of residence time with the two objects $(t=0.408, d f=12, p=0.691$, Fig. $5 \mathrm{~d})$.

The results of the individual recognition test showed that with ongoing trials, voles became familiar with the conspecific, and thus spent less time in close pursuit (Trial: $F_{(3,57)}=7.047, p<0.001$; Groups: $F_{(2,19)}=1.468, p=0.255$,
Fig. 5e) and engaged in fewer investigations of the conspecific (Trial: $F_{(3,57)}=49.426, p<0.001$; Groups; $\mathrm{F}_{(2,19)}=$ $1.212, p=0.320$, Fig. 5f). During Trial 3, when voles exposed to the same young vole for the third time, it was revealed that acetate supplementation rescued the phenotype of Control voles. In Trial 3, both the Control and CP- 
Ace groups exhibited lower times of close pursuit $\left(F_{(2,18)}=\right.$ 4.093, $p=0.034$, Fig. 5e) and lower frequency of investigation $\left(F_{(2,18)}=4.526, p=0.026\right.$, Fig. $\left.5 f\right)$ when compared to the CP-PBS groups, suggesting impairment in the ability of the CP-PBS group to recognize the conspecific individual. In Trial 4, when a novel individual was introduced, we observed increases in pursuit time and frequency of investigation in all groups, but there were no significant differences across treatment groups (pursuit time: $F_{(2,18)}=1.429$, $p=0.266$, Fig. 5e; frequency of investigation $F_{(2,18)}$ $=0.977, p=0.395$, Fig. 5f).

\section{Supplementation of acetate can alter hippocampal neurochemistry}

To investigate potential mechanisms underlying the differences observed above, we measured the expression of many proteins related to cognition (Table S1). We found several proteins where acetate supplementation was able to reverse changes induced by $\mathrm{CP}$ (Fig. $5 \mathrm{~g}$ ). For example, acetate increased the expression of OT $\left(F_{(2,18)}=3.787\right.$, $p=0.042)$, NeuN $\left(F_{(2,18)}=5.284, p=0.016\right)$, PCNA $\left(F_{(2,18)}=3.586, p=0.049\right)$, and AVP $\left(F_{(2,18)}=6.416\right.$, $p=0.008$, Fig. $5 \mathrm{~g}$ ).

However, acetate supplementation did not completely rescue all aspects of hippocampal protein expression. For example, acetate did not rescue expression of BDNF, which is involved in neurogenesis. BDNF expression was lower in both the CP-PBS and CP-Ace groups when compared with the Control group $\left(F_{(2,18)}=4.693, p=0.023\right.$, Fig. 5g).

Acetate supplementation distinctly upregulated expression of several proteins. For example, the $\mathrm{CP}$-Ace groups exhibited significantly higher expression of $\operatorname{GFAP}\left(F_{(2,18)}=\right.$ 16.537, $p<0.001)$ and the vasopressin receptor (AVP-R1; $F_{(2,18)}=14.177, p<0.001$, Fig. $\left.5 \mathrm{~g}\right)$.

\section{Discussion}

Coprophagy is a widespread behavior among small- and medium-sized mammalian herbivores. This behavior can help to improve the digestive function of herbivorous animals, because many nutrients liberated by the hindgut microbiota can be digested and absorbed by eating feces [42]. Here, we demonstrate that prevention of coprophagy alters the community structure of the gut microbiome and affects many physiological processes and cognitive performance in Brandt's voles. In this study, we are unable to fully disentangle many mechanisms by which $\mathrm{CP}$ may alter the composition and function of the microbiome and downstream effects on host physiology, as hypothesized mechanisms (discussed more below) are all highly interconnected. It does seem that the effects of $\mathrm{CP}$ are at least partially regulated by SCFAs, as we also demonstrate that acetate supplementation can alleviate energy imbalance and improve cognitive performance in animals during $\mathrm{CP}$. We discuss these findings and their implications more below.

\section{$\mathrm{CP}$ alters the host gut microbiota}

In wild animals, the gut microbiota is affected by many environmental factors, including temperature, season, food quality, etc. [43-45]. Early, culture-based studies in laboratory rodents have demonstrated that prevention of coprophagy can alter aspects of the gut microbiome [46]. For example, $\mathrm{CP}$ in rats yielded increases in the abundances of Escherichia coli and Enterococcus and decreases in Lactobacillus [14]. Here, we used high-throughput sequencing to more thoroughly understand the impacts of $\mathrm{CP}$ on the gut microbiome of an herbivorous rodent.

Our experiments revealed that $\mathrm{CP}$ significantly decreased the abundance of the phylum Firmicutes, composed of families like the Clostridiaceae, Lachnospiraceae, and Ruminococcaceae. These families of the Firmicutes are obligate anaerobes, which reduce the oxidative state of the gut [47]. Highly cellulolytic bacterial species such as Ruminococcus flavefaciens are regarded as essential for the microbial breakdown of cellulose in herbivores [48]. In our results, $R$. flavefaciens decreased after $\mathrm{CP}$, which may ultimately reduce cellulose fermentation capacity. Voles experiencing $\mathrm{CP}$ also exhibited significantly lower abundances of Oscillospira, a bacterium has been detected from the human microbiome in association with leanness or lower body mass index in both infants and adults [49-51]. Oscillospira are not fiber degraders but rather produce butyrate by relying on fermentation products produced by other microbes or on sugars liberated from host mucins [52]. Therefore, lower abundances of Oscillospira could contribute to the energy imbalance in voles that are unable to engage in coprophagy.

We also predicted microbiota function from our taxonomic inventories and found that $\mathrm{CP}$ decreased predicted abundances of genes associated with glycolysis and amino acid metabolism. These results suggest that the genes associated with glucose metabolism decrease in abundance after $\mathrm{CP}$, which may reduce the energy supply and metabolic status of intestinal epithelial cells and systemic tissues. We also detected that CP causes decreases in the concentrations of numerous SCFAs, especially acetic acid, propionic acid, and butyric acid. These decreases in SCFA could be driven by reductions in the abundances of known fiber degraders, such as Ruminococcus. In addition, it is worth noting that our inventories only investigate relative abundances of microbial taxa. It would be interesting to study how CP alters the absolute abundance, or density of microbial cells in the gut. 
Numerous mechanisms may underlie the alterations to the gut microbiome induced by $\mathrm{CP}$. First, coprophagy may act to re-inoculate animals with their own microbiome, as fecal matter contains a dense community of live microbes. Studies comparing the microbial communities across gut regions of herbivorous rodents actually show the highest similarity between feces and the stomach, suggesting that the fecal community is reinoculated through coprophagy [53]. Nutritional effects of coprophagy may also underlie these observed changes. Diet composition has been shown to rapidly alter the gut microbiome [54]. Here, voles in the control and coprophagy groups were likely consuming different macronutrient compositions, as the fecal material generally has higher protein content and lower fiber content than ingested food material [55]. Last, in addition to nutrient composition, the amount of food consumed can alter the gut microbiota [56]. In our study, voles that were not allowed to engage in coprophagy consumed significantly more food material than the control group (though this increase in food intake was not sufficient for animals to maintain body mass). In the future, experimental methods such as artificial microbial inoculations, feeding of autoclaved feces, or other techniques will be required to disentangle the mechanisms by which $\mathrm{CP}$ alter the microbiome.

\section{Coprophagy affects host energy metabolism}

Our results showed that $\mathrm{CP}$ resulted in increased food intake, yet reduced body mass. These results are similar to previous studies in rats [57, 58], meadow voles (Microtus pennsylvanicus), and woodland voles (Microtus pinetorum) [59]. The soft feces excreted by rodents are rich in vitamins, amino acids, peptides, and a variety of nutrients that are not digested and absorbed by the distal digestive tract [11], and experiments show that the concentrations of these nutrients in rodent feces increases significantly as a result of $\mathrm{CP}$ $[58,60]$. Our measurements of gut morphology showed that the $\mathrm{CP}$ group exhibited shorter intestinal villi compared with the control and sham-collar groups, which is a common physiological response to malnutrition [61]. Also, voles in the CP group exhibited RMR that were $28.2 \%$ lower than the other groups. Some studies demonstrate that rodents depress metabolic rates when under calorie restriction, though results here are equivocal across studies [62]. In our study, CP animals consumed more food, yet these increases were insufficient to compensate for nutritional deficiencies, as these animals still lost $\sim 20 \%$ of their body mass. These results suggest that the prevention of coprophagy likely limits voles' access to bacteria-produced nutrients, either due to the inability to consume feces, or though changes to the composition and function of the cecal microbiome. In fact, even with sufficient calories, an imbalance of amino acids or vitamins can cause decreases in rodent body mass [63], and so the lack of some specific microbial products may result in modifications to host physiology.

\section{CP affects vole's cognitive performance and neurochemistry}

We found that $\mathrm{CP}$ significantly altered the cognitive performance of voles. After CP, Brandt's vole exhibited decreased cognitive performance, as they spent less time in the "food arm" in the y-maze. In addition, voles exposed to CP also showed impairment in the novel objects recognition and individual recognition tests. In the wild, memory and cognition are necessary for the life and reproduction [64]. Spatial memory can be especially important for localizing food resources [65]. In addition, social cognition may also affect mating choices [66, 67], and cooperative breeding [68].

These effects may be mediated by the gut microbiome and microbial metabolites via the vagus nerve and neuroendocrine system [21]. Specific brain regions, such as the hypothalamus and hippocampus, participate in regulating learning, memory, and social behavior through neurotransmitters like 5-HT and DA, and the loss of these monoaminergic substances is among the most important neurochemical process leading to cognitive impairment [69-72]. Our results showed that the CP group had lower concentrations of 5-HT and its metabolite 5-HIAA in the hippocampus (though it should be noted that our assays represent total tissue levels, and not levels at synapses or specific cell types). These differences may be caused by alterations to the microbiome, as numerous commensal bacteria influence the production of precursors to these neurotransmitters $[73,74]$. In addition, we found changes in the levels of DA and the metabolite DOPAC in the hippocampus. Although not all monoamine transmitters are reduced, all results highlight that absence or changes of the gut microbiota by $\mathrm{CP}$ affect neurotransmitter systems.

In addition, changes to the levels of central neurohormones may underly these changes in cognitive performance. For example, we found that $\mathrm{CP}$ reduced expression levels of AVP and OT. Both of these neurohormones play important roles in the regulation of central nervous functions related to spatial learning, social cognition, and social behavior [75-77]. Numerous studies have shown that AVP and its analogs can improve learning and cognitive performance [78, 79]. For example, injection of synthetic AVP improves the performance of rats in active and short-term olfactory memory tests [80]. Administration of AVP can facilitate the consolidation and retrieval processes of both active memory [81] and reference memory in a radial maze $[82,83]$. OT injected into the lateral part of the septal brain area has been reported to improve juvenile recognition abilities in adult male rats $[84,85]$. Both AVP and OT injected centrally 
reduced social aggression and increased affiliative behavior in rodents [86-88]. In our experiment, the CP group performed poorly in the Y-maze and for social cognition, and exhibited decreased AVP and OT levels in hippocampus. Overall, microbial and/or nutritional alterations resulting from CP lead to alterations in neurochemistry that may result in impaired cognitive performance.

\section{Acetate supplementation reverses changes induced by cophrophagy prevention}

The gut microbiome elicits many physiological effects on their hosts through the production of fermentation products, including SCFAs [89]. For example, SCFAs affect the energy metabolism of the host [90] and transcriptional regulation within the central nervous system [91, 92]. In our study, CP altered the composition and function of the gut microbiome and decreased the total concentration of SCFAs in the cecum. Interestingly, supplementation with acetate fully or partially reversed numerous changes induced by $\mathrm{CP}$.

First, supplementation of acetic acid rescued the structure of the gut microbiome, such that the relative abundances of numerous microbial phyla were similar between control and acetate-supplemented animals. These changes to microbiome structure also yielded improvements in function, as the concentrations of other SCFAs, such as propionate and butyrate, were similar between control and acetatesupplemented animals. Acetic acid and other SCFAs are often used by intestinal epithelial cells as nutrients [93, 94]. Thus, the increase in their concentrations may alter the physical and chemical properties of the digestive tract and thereby restructure the composition of the gut microbiome. In addition, the gut microbiome engages in cross-feeding, where products of one microbe can be used by another. For example, some gut microbes can utilize extracellular acetate to produce butyrate [95]. These interactions may explain how the supplementation of acetate reversed changes to the microbiome induced by $\mathrm{CP}$.

Next, experimental acetate supplementation rescued alterations to animal body mass, and partially returned food intake levels to those of control animals. There may be several mechanistic pathways to explain this relationship. First, acetate itself and the other SCFAs that were induced by acetate supplementation serve as nutrients to the host [93, 94], and so supplementing animals with this SCFA may directly improve their energy balance. In addition, supplementations with SCFAs may increase the nutritional yield from ingested food. For example, SCFAs can increase the secretion of pancreatic enzymes [96], and expand intestinal blood vessels and increase intestinal blood flow [97], both of which could contribute to increased nutrient absorption. So, engaging in coprophagy behavior might increase energy absorptive capacity by increasing acetate concentration and their downstream physiological effects, helping animals to maintain energy balance.

A wide range of compounds generated by gut microbiota, including SCFAs, have direct or indirect effects on the brain and behavior, including the expression of neuropeptides [91]. Intracellular SCFAs, including acetate, can inhibit the activity of histone deacetylase, promoting the hyperacetylation of histones, and thereby altering gene expression. Studies in rodents have also shown that inhibiting histone deacetylase can enhance cognitive function in fear, anxiety, and trauma $[98,99]$. In our results, supplementation of acetate decreased the expression CRF and CRF receptor in hippocampus to better match expression levels of control animals. Our results are consistent with another study in mice where SCFA supplementation decreased expression of CRF and CRF-R in the hypothalamus [100].

Acetate supplementation also increased expression numerous neuropeptides to match that of control animals. For example, acetate supplementation increased expression of AVP, OT, and their receptors. These neuropeptide hormones are related to the improvement of social behavior and cognitive ability [101-103]. For example, male rats repeatedly exposed to females typically spend less time investigating these conspecifics. Injection with AVP facilitates social memory formation, resulting in decreased investigation, while injection of a vasopressor antagonist impairs this memory formation [104]. Similar studies have demonstrated a role for OT in social memory formation [105]. In our study, CP reduced AVP and OT expression, and these animals also exhibited impaired social memory. These alterations were all reversed by acetate supplementation, suggesting that coprophagy and the downstream physiological effects are important for maintenance of brain neurochemistry and social memory formation.

Last, we observed that acetate supplementation rescued expression levels of NeuN protein to match that of control animals. This protein is only expressed in neuronal cells, and can be used as a marker of newly generated neurons [106]. In rats exposed to a $15 \%$ calorie restriction, NeuNexpressing cells were significantly increased in the hippocampus, and also exhibited enhanced spatial learning and memory [101]. It is interesting that in our study, voles subjected to CP experienced nutritional limitations (perhaps similar to calorie restriction), yet exhibited lower NeuN expression and cognitive performance. These discrepancies suggest that the alterations to cognitive performance in our study are not due solely to nutritional limitation.

\section{Conclusions}

The current study suggests that $\mathrm{CP}$ causes a significant disturbance to the gut bacterial community, which can 
impact animal energy balance and downstream behaviors. Thus, engaging in the behavior of coprophagy helps animals to host a stable gut microbiome, recover microbially produced metabolites, and maintain neurochemistry and cognitive performance. To our knowledge, this study is among the first to use high-depth sequencing approaches to demonstrate the impact of coprophagy on the gut microbiota, and to demonstrate downstream effects on cognitive performance in a wild animal. Further, we demonstrated that microbial products, such as acetate, are at least partially responsible for the changes induced by CP. Supplementation with acetate rescued aspects of microbiome structure, brain neurochemistry, and cognitive performance. Future experiments should work to disentangle the many interacting mechanisms that may underlie these results.

\section{Data availability}

Raw sequence data are deposited in the NCBI Sequence Read Archive under accession PRJNA588029.

Acknowledgements We appreciate the very helpful and constructive comments and suggestions from the three anonymous reviewers and the editor for improving the manuscript. We thank Jianfeng Wang and Bin Chen from Beijing Nebula Medical Laboratory Co., Ltd for helps in $16 \mathrm{~S}$ rRNA gene sequencing and analyses. We thank all the members of Animal Physiological Ecology Group for their help in the experiments and discussions.

Funding This research was supported by the National Natural Science Foundation of China (Nos. 31770440 and 31772461), and the Beijing Natural Science Foundation (5172024).

Author contributions Conceptualization: $\mathrm{X}-\mathrm{YZ}, \mathrm{T}-\mathrm{BB}$, and D-HW; experiment: T-BB, JW, and S-JT; writing: T-BB, X-YZ, D-HW, and $\mathrm{KDK}$; supervision and project management: D-HW and X-YZ; funding acquisition: D-HW and X-YZ.

\section{Compliance with ethical standards}

Conflict of interest The authors declare that they have no conflict of interest.

Ethical approval Our experimental protocol has been approved by the Ethics Committee of the Institute of Zoology of Chinese Academy of Sciences.

Publisher's note Springer Nature remains neutral with regard to jurisdictional claims in published maps and institutional affiliations.

\section{References}

1. Sommer F, Backhed F. The gut microbiota-masters of host development and physiology. Nat Rev Microbiol. 2013;11: 227-38.

2. Heijtz RD, Wang S, Anuar F, Qian Y, Bjorkholm B, Samuelsson $A$, et al. Normal gut microbiota modulates brain development and behavior. Proc Natl Acad Sci USA. 2011;108:3047-52.
3. Al-Asmakh M, Anuar F, Zadjali F, Rafter J, Pettersson S. Gut microbial communities modulating brain development and function. Gut Microbes. 2012;3:366-73.

4. Den Besten G, van Eunen K, Groen AK, Venema K, Reijngoud D-J, Bakker BM. The role of short-chain fatty acids in the interplay between diet, gut microbiota, and host energy metabolism. J Lipid Res. 2013;54:2325-40.

5. Mackie RI. Mutualistic fermentative digestion in the gastrointestinal tract: diversity and evolution. Integr Comp Biol. 2002;42:319-26.

6. Bergman EJ. Energy contributions of volatile fatty acids from the gastrointestinal tract in various species. Physiol Rev. 1990;70: 567-90.

7. Justice KE, Smith FA. A model of dietary fiber utilization by small mammalian herbivores, with empirical results for Neotoma. Am Naturalist. 1992;139:398-416.

8. Stevens CE, Hume ID. Comparative physiology of the vertebrate digestive system. Cambridge University Press; UK, 2004.

9. Matsuzawa T, Nakata M, Tsushima M. Feeding and excretion in the Afghan pika (Ochotona rufescens rufescens), a new laboratory animal. Lab Anim. 1981;15:319-22.

10. Liu QS, Wang DH. Effects of diet quality on phenotypic flexibility of organ size and digestive function in Mongolian gerbils (Meriones unguiculatus). J Comp Physiol B. 2007;177:509-18.

11. Pei YX, Wang DH, Hume ID. Selective digesta retention and coprophagy in Brandt's vole (Microtus brandti). J Comp Physiol B. $2001 ; 171: 457-64$.

12. Savage DC. Gastrointestinal microflora in mammalian nutrition. Annu Rev Nutr. 1986;6:155-78.

13. Klaasen HLBM, Koopman JP, Scholten PM, Van DBME, Theeuwes AGM. Effect of preventing coprophagy on colonisation by segmented filamentous bacteria in the small bowel of mice. Microb Ecol Health Dis. 1990;3:99-103.

14. Fitzgerald RJ, Gustafsson BE, McDaniel EG. Effects of coprophagy prevention on intestinal microflora in rats. $J$ Nutr. 1964;84:155-60.

15. Sarkar A, Harty S, Lehto SM, Moeller AH, Dinan TG, Dunbar RIM, et al. The microbiome in psychology and cognitive neuroscience. Trends Cogn Sci. 2018;22:611-36.

16. Sandhu KV, Eoin Sherwin HS, Catherine S, Timothy GD, John FC. Feeding the microbiota-gut-brain axis: diet, microbiome, and neuropsychiatry. Transl Res. 2017;179:223-44.

17. Ling Z, Cheng Y, Li L. Gut microbiota depletion from early adolescence in mice: Implications for brain and behavior. Brain Behav Immun. 2015;48:165-73.

18. Sampson TR, Mazmanian SK. Control of brain development, function, and behavior by the microbiome. Cell Host Microbe. 2015;17:565-76.

19. Borovikova LV, Ivanova S, Zhang M, Yang H, Botchkina GI, Watkins LR, et al. Vagus nerve stimulation attenuates the systemic inflammatory response to endotoxin. Nature. 2000;405:458-62.

20. Wang X, Wang BR, Zhang XJ, Xu Z, Ding YQ, Ju G. Evidences for vagus nerve in maintenance of immune balance and transmission of immune information from gut to brain in STMinfected rats. World J Gastroenterol. 2002;8:540-5.

21. Bravo JA, Forsythe P, Chew MV, Escaravage E, Savignac HM, Dinan TG, et al. Ingestion of Lactobacillus strain regulates emotional behavior and central GABA receptor expression in a mouse via the vagus nerve. Proc Natl Acad Sci USA. 2011;108:16050-5.

22. Tremaroli V, Backhed F. Functional interactions between the gut microbiota and host metabolism. Nature. 2012;489:242-9.

23. Ziętak M, Kovatcheva-Datchary P, Markiewicz LH, Ståhlman M, Kozak LP, Bäckhed F. Altered microbiota contributes to reduced diet-induced obesity upon cold exposure. Cell Metab. 2016;23:1216-23. 
24. Bo TB, Zhang XY, Wen J, Deng K, Qin XW, Wang DH. The microbiota-gut-brain interaction in regulating host metabolic adaptation to cold in male Brandt's voles (Lasiopodomys brandtii). ISME J. 2019;13:3037-53.

25. Laukens D, Brinkman BM, Raes J, De Vos M, Vandenabeele P. Heterogeneity of the gut microbiome in mice: guidelines for optimizing experimental design. FEMS Microbiol Rev. 2016; 40:117-32.

26. Haim A, Izhaki I. The ecological significance of resting metabolic rate and non-shivering thermogenesis for rodents. J Therm Biol. 1993;18:71-81.

27. Burton T, Killen SS, Armstrong JD, Metcalfe NB. What causes intraspecific variation in resting metabolic rate and what are its ecological consequences?. Proc R Soc B: Biol Sci. 2011;278: 3465-73.

28. Zhang XY, Sukhchuluun G, Bo TB, Chi QS, Yang JJ, Wang $\mathrm{DH}$, et al. Huddling remodels gut microbiota to reduce energy requirements in a small mammal species during cold exposure. Microbiome. 2018;6:103.

29. Montiglio PO, Garant D, Thomas D, Reale D. Individual variation in temporal activity patterns in open-field tests. Anim Behav. 2010;80:905-12.

30. Yao W, Liu W, Deng K, Wang Z, Wang DH, Zhang XY. GnRH expression and cell proliferation are associated with seasonal breeding and food hoarding in Mongolian gerbils (Meriones unguiculatus). Horm Behav. 2019;112:42-53.

31. Lieberwirth C, Liu Y, Jia X, Wang Z. Social isolation impairs adult neurogenesis in the limbic system and alters behaviors in female prairie voles. Horm Behav. 2012;62:357-66.

32. Cardiff RD, Miller CH, Munn RJ. Manual hematoxylin and eosin staining of mouse tissue sections. Cold Spring Harb Protoc. 2014;6:073411.

33. Rueden CT, Schindelin JE, Hiner MC, DeZonia BE, Walter AE, Arena ET, et al. ImageJ2: ImageJ for the next generation of scientific image data. BMC Bioinform. 2017;18:529.

34. Chiu K, Lau WM, Lau HT, So KF, Chang RCC. Microdissection of rat brain for RNA or protein extraction from specific brain region. J Vis Exp. 2017;7:269.

35. Jia M, Meng F, Smerin SE, Xing G, Zhang L, Su DM, et al. Biomarkers in an animal model for revealing neural, hematologic, and behavioral correlates of PTSD. J Vis Exp. 2012;68:3361.

36. Herlemann DP, Labrenz M, Jürgens K, Bertilsson S, Waniek JJ, Andersson AF. Transitions in bacterial communities along the $2000 \mathrm{~km}$ salinity gradient of the Baltic Sea. ISME J. 2011;5:1571-9.

37. Douglas GM, Maffei VJ, Zaneveld J R, Yurgel SN, Langille MGI. PICRUSt2 for prediction of metagenome functions. Nature Biotechnology. 2020; D1:1-5.

38. Parks DH, Tyson GW, Hugenholtz P, Beiko RG. STAMP: statistical analysis of taxonomic and functional profiles. Bioinformatics. 2014;30:3123-4.

39. Cartmell J, Salhoff CR, Perry KW, Monn JA, Schoepp DD. Dopamine and 5-HT turnover are increased by the mGlu2/3 receptor agonist LY379268 in rat medial prefrontal cortex, nucleus accumbens and striatum. Brain Res. 2000;887:378-84.

40. Kim KI, van de Wiel MA. Effects of dependence in highdimensional multiple testing problems. BMC Bioinform. 2008;9:114

41. Segata N, Izard J, Waldron L, Gevers D, Miropolsky L, Huttenhower $\mathrm{C}$, et al. Metagenomic biomarker discovery and explanation. Genome Biol. 2011;12:R60.

42. Hörnicke H, Björnhag G. Coprophagy and related strategies for digesta utilization. In: Ruckebusch Y., Thivend P. (eds) Digestive physiology and metabolism in ruminants. Published by MTP Press Limited, England, 1980;34:707-30.
43. Bäckhed F, Ding H, Wang T, Hooper LV, Koh GY, Gordon JI, et al. The gut microbiota as an environmental factor that regulates fat storage. Proc Natl Acad Sci USA. 2004;101:15718-23.

44. Li H, Qu J, Li T, Wirth S, Zhang Y, Li X, et al. Diet simplification selects for high gut microbial diversity and strong fermenting ability in high-altitude pikas. Appl Microbiol Biotechnol. 2018;102:6739-51.

45. Sommer F, StHlman M, Ilkayeva O, Arnemo J, Kindberg J, Josefsson $J$, et al. The Gut Microbiota Modulates Energy Metabolism in the hibernating brown bear Ursus arctos. Cell Rep. 2016;14:1655-61.

46. Klaasen HL, Koopman JP, Poelma FG, Beynen AC. Intestinal, segmented, filamentous bacteria. FEMS Microbiol Rev. 1992;8: 165-79.

47. Sagheddu V, Patrone V, Miragoli F, Puglisi E, Morelli L. Infant early gut colonization by Lachnospiraceae: high frequency of Ruminococcus gnavus. Front Pediatr. 2016;4:57.

48. Præsteng KE, Pope PB, Cann IKO, Mackie RI, Mathiesen SD, Sundset MA, et al. Probiotic dosing of Ruminococcus flavefaciens affects rumen microbiome structure and function in reindeer. Micro Ecol. 2013;66:840-9.

49. Tims S, Derom C, Jonkers DM, Vlietinck R, Saris WH, Zoetendal EG, et al. Microbiota conservation and BMI signatures in adult monozygotic twins. ISME J. 2013;7:707-17.

50. Goodrich J, DiRienzi S, Poole A, Koren O, Walters W, Caporaso J, et al. Conducting a microbiome study. Cell. 2014;158:250-62.

51. Verdam FJ, Fuentes S, de Jonge C, Zoetendal EG, Erbil R, Rensen SS, et al. Human intestinal microbiota composition is associated with local and systemic inflammation in obesity. Obesity. 2013;21:E607-15.

52. David LA, Maurice C, Carmody RN, Gootenberg DB, Button JE, Fischbach MA, et al. Diet rapidly and reproducibly alters the human gut microbiome. Nature. 2014;505:559-63.

53. Kohl KD, Miller AW, Marvin JE, Mackie R, Dearing MD. Herbivorous rodents (Neotoma spp.) harbour abundant and active foregut microbiota. Environ Microbiol. 2014;16:2869-78.

54. Carmody RN, Gerber GK, Luevano JM, Gatti DM, Somes L, Turnbaugh PJ, et al. Diet dominates host genotype in shaping the murine gut microbiota. Cell Host Microbe. 2015;17:72-84.

55. Liu QS, Li JY, Wang DH. Ultradian rhythms and the nutritional importance of caecotrophy in captive Brandt's voles (Lasiopodomys brandtii). J Comp Physiol B. 2007;177:423-32.

56. Zhang C, Li S, Yang L, Huang P, Li W, Zhao L, et al. Structural modulation of gut microbiota in life-long calorie-restricted mice. Nat Commun. 2013;4:2163.

57. Cree TC, Wadley DM, Marlett JA. Effect of preventing coprophagy in the rat on neutral detergent fiber digestibility and apparent calcium absorption. J Nutr. 1986;116:1204-8.

58. Sukemori S, Ikeda S, Kurihara Y, Ito S. Amino acid, mineral and vitamin levels in hydrous faeces obtained from coprophagyprevented rats. J Anim Physiol Anim Nutr. 2003;87:213-20.

59. Cranford JA, Johnson EO. Effects of coprophagy and diet quality on two microtine rodents (Microtus pennsylvanicus and Microtus pinetorum). J Mammal. 1989;70:494-502.

60. Stillings BR, Hackler LR. Effect of coprophagy on protein utilization in the rat. J Nutr. 1966;90:19-24.

61. Dunel-Erb S, Chevalier C, Laurent P, Bach A, Decrock F, Le Maho Y. Restoration of the jejunal mucosa in rats refed after prolonged fasting. Comp Biochem Physiol Part A: Mol Integr Physiol. 2001;129:933-47.

62. Merry BJ. Molecular mechanisms linking calorie restriction and longevity. Int J Biochem Cell Biol. 2002;34:1340-54.

63. Sauberlich HJ. Amino acid imbalance as related to methionine, isoleucine, threonine and tryptophan requirement of the rat or mouse. J Nutr. 1956;59:353-70. 
64. Yan L, Sun X, Wang Z, Song M, Zhang Z. Regulation of social behaviors by p-Stat 3 via oxytocin and its receptor in the nucleus accumbens of male Brandt's voles (Lasiopodomys brandtii). Horm Behav. 2020;119:104638.

65. Janson $\mathrm{CH}$. Experimental evidence for spatial memory in foraging wild capuchin monkeys, Cebus apella. Anim Behav. 1998;55:1229-43.

66. Phelps SM, Rand AS, Ryan MJ. A cognitive framework for mate choice and species recognition. Am Naturalist. 2006;167:28-42.

67. Parker JT, Rodriguez N, Lawal B, Delevan CJ, \& Bamshad M. Mating increases male's interest in other females: a cognitive study in socially monogamous prairie voles (microtus ochrogaster). Behav Processes. 2011;88:127-34.

68. Burkart JM, Van Schaik CP. Cognitive consequences of cooperative breeding in primates?. Anim Cognition. 2010;13:1-19.

69. Luine V, Hearns M. Spatial memory deficits in aged rats: contributions of the cholinergic system assessed by ChAT. Brain Res. 1990;523:321-4.

70. Bowman RE, Beck KD, Luine VN. Chronic stress effects on memory: sex differences in performance and monoaminergic activity. Hormones Behav. 2003;43:48-59.

71. Deng XH, Liu Y, Chen ZG. Memory-based evolutionary game on small-world network with tunable heterogeneity. Phys A: Stat Mech its Appl. 2010;389:5173-81.

72. Malberg JE, Eisch AJ, Nestler EJ, Duman RS. Chronic antidepressant treatment increases neurogenesis in adult rat hippocampus. J Neurosci. 2000;20:9104-10.

73. Vadder FD, Grasset E, Holm LM, Karsenty G, Macpherson AJ, Bäckhed F, et al. Gut microbiota regulates maturation of the adult enteric nervous system via enteric serotonin networks. Proc Natl Acad Sci USA. 2018;115:6458-63.

74. O'Mahony SM, Clarke G, Borre YE, Dinan TG, Cryan JF. Serotonin, tryptophan metabolism and the brain-gut-microbiome axis. Behav Brain Res. 2015;277:32-48.

75. Liebsch G, Wotjak CT, Landgraf R, Engelmann M. Septal vasopressin modulates anxiety-related behavior in rats. Neurosci Lett. 1996;217:101-4.

76. Cilz NI, Cymerblit-Sabba A, Young WS. Oxytocin and vasopressin in the rodent hippocampus. Genes Brain Behav. 2019;18: e12535.

77. Johnson ZV, Young LJ. Oxytocin and vasopressin neural networks: Implications for social behavioral diversity and translational neuroscience. Neurosci Biobehav Rev. 2017;76: 87-98.

78. Hamburger-Bar, Klein R,A, Belmaker RH. The effect of chronic vs. acute injection of vasopressin on animal learning and memory. Peptides. 1985;6:23-5.

79. Bluthe RM, Koob GF, Dantzer R. Hypertonic saline mimics the effects of vasopressin and social recognition in rats. Behav Pharm. 1991;2:513-6.

80. Bohus B, Urban I, Vanwimersmagreidanus $\mathrm{T}$, Dewied $\mathrm{D}$. Opposite effects of oxytocin and vasopressin on avoidance behavior and hippocampal theta rhythm in the rat. Neuropharmacology. 1978;17:239-47.

81. Metzger D, Alescio-Lautier B, Bosler O, Devigne C, SoumireuMourat B. Effect of changes in the intrahippocampal vasopressin on memory retrieval and relearning. Behav Neural Biol. 1993;59:29-48.

82. Dietrich A, Allen JD. Vasopressin and memory. II. Lesions to the hippocampus block the memory enhancing effects of AVP49 in the radial maze. Behav Brain Res. 1997;87:201-8.

83. Yang C, Zhang X, Gao J, Wang M, Yang Z. Arginine vasopressin ameliorates spatial learning impairments in chronic cerebral hypoperfusion via V1a receptor and autophagy signaling partially. Transl Psychiatry. 2017;7:e1174.
84. Popik P, Van Ree JM. Long-term facilitation of social recognition in rats by vasopressin related peptides: a structure-activity study. Life Sci. 1992;50:567-72.

85. Lee SY, Park SH, Chung C, Kim JJ, Choi SY, Han JS. Oxytocin protects hippocampal memory and plasticity from uncontrollable stress. Sci Rep. 2015;5:18540.

86. Burkett JP, Andari E, Johnson ZV, Curry DC, de Waal FBM, Young LJ. Oxytocin-dependent consolation behavior in rodents. Science. 2016;351:375-8.

87. Gobrogge KL, Jia X, Liu Y, Wang Z. Neurochemical mediation of affiliation and aggression associated with pair-bonding. Biol Psychiatry. 2017;81:231-42.

88. Freeman AR, Hare JF, Anderson WG, Caldwell HK. Effects of arginine vasopressin on Richardson's ground squirrel social and vocal behavior. Behav Neurosci. 2018;132:34.

89. Kohl KD, Carey HV. A place for host-microbe symbiosis in the comparative physiologist's toolbox. J Exp Biol. 2016;219: 3496-504.

90. Turnbaugh PJ, Ley RE, Mahowald MA, Magrini V, Mardis ER, Gordon JI. An obesity-associated gut microbiome with increased capacity for energy harvest. Nature. 2006;444:1027-31.

91. Stilling RM, Dinan TG, Cryan JF. Microbial genes, brain \& behavior-epigenetic regulation of the gut-brain axis. Genes Brain Behav. 2014;13:69-86.

92. Luczynski P, Whelan SO, O'Sullivan C, Clarke G, Shanahan F, Dinan TG, et al. Adult microbiota-deficient mice have distinct dendritic morphological changes: differential effects in the amygdala and hippocampus. Eur J Neurosci. 2016;44: 2654-66.

93. Zambell KL, Fitch MD, Fleming SE. Acetate and butyrate are the major substrates for de novo lipogenesis in rat colonic epithelial cells. J Nutr. 2003;133:3509-15.

94. Donohoe DR, Garge N, Zhang X, Sun W, O'Connell TM, Bunger MK, et al. The microbiome and butyrate regulate energy metabolism and autophagy in the mammalian colon. Cell Metab. 2011;13:517-26.

95. Duncan SH, Holtrop G, Lobley GE, Calder AG, Stewart CS, Flint HJ. Contribution of acetate to butyrate formation by human faecal bacteria. Br J Nutr. 2004;91:915-23.

96. Harada E, Kato S. Effect of short-chain fatty acids on the secretory response of the ovine exocrine pancreas. Am J Physiol. 1983;244:G284-90.

97. Kvietys PR, Granger DN. Effect of volatile fatty acids on blood flow and oxygen uptake by the dog colon. Gastroenterology. 1981;80:962-9.

98. Whittle N, Singewald N. HDAC inhibitors as cognitive enhancers in fear, anxiety and trauma therapy: where do we stand? Biochemical Soc Trans. 2014;42:569.

99. Singewald N, Schmuckermair C, Whittle N, Holmes A, Ressler KJ. Pharmacology of cognitive enhancers for exposure-based therapy of fear, anxiety and trauma-related disorders. Pharm Ther. 2015;149:150-90.

100. Van de Wouw M, Boehme M, Lyte JM, Wiley N, Strain C, Cryan JF, et al. Short-chain fatty acids: microbial metabolites that alleviate stress-induced brain-gut axis alterations. J Physiol. 2018;596:4923-44.

101. Kaptan Z, Akgün-Dar K, Kapucu A, Dedeakayoğulları H, Batu Ş, Üzüm G. Long term consequences on spatial learningmemory of low-calorie diet during adolescence in female rats; hippocampal and prefrontal cortex BDNF level, expression of NeuN and cell proliferation in dentate gyrus. Brain Res. 2015;1618:194-204.

102. Engelmann M, Wotjak CT, Neumann I, Ludwig M, Landgraf R. Behavioral consequences of intracerebral vasopressin and oxytocin: focus on learning and memory. Neurosci Biobehav Rev. 1996;20:341-58. 
103. Song Z, Larkin TE, Malley MO, Albers HE. Oxytocin (OT) and arginine-vasopressin (AVP) act on OT receptors and not AVP V1a receptors to enhance social recognition in adult Syrian hamsters (Mesocricetus auratus). Hormones Behav. 2016;81:20-27.

104. Dantzer R, Koob GF, Bluthe RM, Le Moal M. Septal vasopressin modulates social memory in male rats. Brain Res. $1988 ; 457: 143-7$.
105. Lukas M, Toth I, Veenema AH, Neumann ID. Oxytocin mediates rodent social memory within the lateral septum and the medial amygdala depending on the relevance of the social stimulus: male juvenile versus female adult conspecifics. Psychoneuroendocrinology. 2013;38:916-26.

106. Wolf HK, Buslei R, Schmidt-Kastner R, Schmidt-Kastner PK, Pietsch T, Blümcke I, et al. NeuN: a useful neuronal marker for diagnostic histopathology. J Histochem Cytochem. 1996;44:1167-71. 